\title{
SCATTERING THEORY FOR A CLASS OF NON-SELFADJOINT EXTENSIONS OF SYMMETRIC OPERATORS
}

\author{
Kirill D. Cherednichenko \\ Department of Mathematical Sciences, \\ University of Bath, \\ Claverton Down, Bath BA2 7AY, U.K. \\ K. Cherednichenko@bath.ac.uk \\ Alexander V. Kiselev \\ Departamento de Física Matemática, \\ Instituto de Investigaciones en Matemáticas Aplicadas y en Sistemas, \\ Universidad Nacional Autónoma de México, \\ C.P. 04510, México D.F. \\ and \\ International Research Laboratory "Multiscale Model Reduction", \\ Ammosov North-Eastern Federal University, \\ Yakutsk, Russia \\ alexander.v.kiselev@gmail.com \\ Luis O. Silva \\ Departamento de Física Matemática, \\ Instituto de Investigaciones en Matemáticas Aplicadas y en Sistemas, \\ Universidad Nacional Autónoma de México, \\ C.P. 04510, México D.F. \\ silva@iimas.unam.mx
}

To the fond memory of Professor Boris Pavlov

\begin{abstract}
This work deals with the functional model for a class of extensions of symmetric operators and its applications to the theory of wave scattering. In terms of Boris Pavlov's spectral form of this model, we find explicit formulae for the action of the unitary group of exponentials corresponding to almost solvable extensions of a given closed symmetric operator with equal deficiency indices. On the basis of these formulae, we are able to construct wave operators and derive a new representation for the scattering matrix for pairs of such extensions in both self-adjoint and nonself-adjoint situations.
\end{abstract}

\section{Introduction}

Over the last eighty years or so, the subject of the mathematical analysis of waves interacting with obstacles and structures ("scattering theory") has served as one of the most impressive examples of bridging abstract mathematics and applications to physics, which in turn motivated the

Mathematics Subject Classification (2010): 47A45 34L25 81Q35

Keywords: Functional model; Extensions of symmetric operators; Boundary triples; Scattering theory 
development of new mathematical techniques. The pioneering works of von Neumann 69, 70] and his contemporaries during 1930-1950, on the mathematical foundations of quantum mechanics, fuelled the interest of mathematical analysts to formulating and addressing the problems of direct and inverse wave scattering in a rigorous way.

The foundations of the modern mathematical scattering theory were laid by Friedrichs, Kato and Rosenblum [29, 71, 22] and subsequently by Birman and Kreŭ [5], Birman [4, Kato and Kuroda [30] and Pearson [54. For a detailed exposition of this subject, see [55, 73].

The direct and inverse scattering on the infinite and semi-infinite line was extensively studied using the classical integral-operator techniques by Borg [7, 8, Levinson [41, Krein [36, 37, 38, Gel'fand and Levitan 23, Marchenko [45, Faddeev [20, 21, Deift and Trubowitz 15. In this body of work, the crucial role is played by the classical Weyl-Titchmarsh $m$-coefficient.

In the general operator-theoretic context, the $m$-coefficient is generalised to both the classical Dirichlet-to-Neumann map (in the PDE setting; cf. also [3]), and to the so-called $M$-operator, which takes the form of the Weyl-Titchmarsh $M$-matrix in the case of symmetric operators with equal deficiency indices. This has been exploited extensively in the study of operators, self-adjoint and non-selfadjoint alike, through the works in Ukraine (brought about by the influence of M.Kreinn) on the theory of boundary triples and the associated $M$-operators (Gorbachuk and Gorbachuk [25], Kočuber [32, 33], Derkach and Malamud [17] and further developments) and of the students of Pavlov in St. Petersburg (see e.g. 60, 31, 10]).

A parallel approach, which provides a connection to the theory of dissipative operators, was developed by Lax and Phillips [40, who analysed the direct scattering problem for a wide class of linear operators in the Hilbert space, including those associated with the multi-dimensional acoustic problem outside an obstacle, using the language of group theory (and, indeed, thereby developing the semigroup methods in operator theory). The associated techniques were also termed "resonance scattering" by Lax and Phillips.

By virtue of the underlying dissipative framework, the above activity set the stage for the applications of non-selfadjoint techniques, in particular for the functional model for contractions and dissipative operators by Szökefalvi-Nagy and Foiaş 67, which has shown the special rôle in it of the characteristic function of Livšic [43] and allowed Pavlov [53] to construct a spectral form of the functional model for dissipative operators. The connection between this work and the concepts of scattering theory was uncovered by the famous theorem of Adamyan and Arov [1. In a closely related development, Adamyan and Pavlov [2] established a description for the scattering matrix of a pair of self-adjoint extensions of a symmetric operator (densely or non-densely defined) with finite equal deficiency indices.

Further, Naboko [48] advanced the research initiated by Pavlov, Adamyan and Arov in two directions. Firstly, he generalised Pavlov's construction to the case of non-dissipative operators, and secondly, he provided explicit formulae for the wave operators and scattering matrices of a pair of (in general, non-selfadjoint) operators in the functional model setting. It is remarkable that in this work of Naboko the difference between the so-called stationary and non-stationary scattering approaches disappears.

There exists a wide body of work, carried out in the last thirty years or so, dedicated to the analysis of the scattering theory for general non-selfadjoint operators [44, 66, 68, 59, 63, 61]. These works make a substantial use of functional model techniques in the non-selfadjoint case and provide the most general results, without taking into account the specific features of any particular subclass of operators under consideration. In particular, the paper 63] essentially generalises to the nonselfadjoint case the classical stationary approach to the construction of wave operators [73]. On the other hand, as pointed out above, the study of non-selfadjoint extensions of symmetric operators naturally lends itself to the use of the theory of boundary triples and associated $M$-operators, thus taking advantage of the concrete properties of this subclass. This has been exploited in [60], where a functional model for dissipative and non-dissipative almost solvable extensions of symmetric operators was developed in terms of the theory of boundary triples. This work, however, stops short of the characterisation of the absolutely continuous subspace of the operator considered in 
the "natural" terms associated with boundary triples and $M$-operators ( $c f$. 59, 56, where the concept of the absolutely continuous subspace of a self-adjoint operator is discussed in the most general case). If one bridges this (in fact, very narrow) gap, as we do in Sections 3 , 4 this opens up a possibility to directly apply Naboko's argument [48, which then yields both the explicit expression for wave operators and concise, easily checked sufficient conditions for the existence and completeness of wave operators, formulated in natural terms. What is more, it also yields an explicit expression for the scattering matrix of the problem, formulated in terms of the $M$-operator and parameters fixing the extension.

Our aim in the present work is therefore twofold: first, it is to expose the methodology of functional model in application to the development of scattering theory for non-selfadjoint operators and, second, to apply this methodology to the case of almost solvable extensions of symmetric operators, yielding new, concise and explicit, results in the special and important in applications case. With this aim in mind, we endeavour to extend the approach of Naboko [48, which was formulated for additive perturbations of self-adjoint operators, to the case of both self-adjoint and non-self-adjoint extensions of symmetric operators, under the only additional assumption that this extension is almost solvable, see Section 2 below for precise definitions. Unfortunately, the named assumption is rather restrictive in nature, see Remark 2 below. Still, already the framework of almost solvable extensions allows us to consider direct and inverse scattering problems on quantum graphs, see 14 for an application of abstract results of this paper in the mentioned setting. We also point out that the case we consider proves to be sufficiently generic to allow for a treatment of the scattering problem for models of double porosity in homogenisation, see [12, 13.

The paper is organised as follows. In Section 2 we recall the key points of the theory of boundary triples for extensions of symmetric operators with equal deficiency indices and introduce the associated $M$-operators, following mainly [17] and 60. In Section 3 we derive formulae for the resolvents of the family of extensions $A_{\varkappa}$ parametrised by operators $\varkappa$ in the boundary space, in terms of the so-called characteristic function of a fixed element of the family. These formulae are then employed in Section 4 to derive the functional model for the above family of extensions. The material of Sections 3 and 4 closely follows the approach of 60] and is based on the much more general facts of e.g. [48, 44, 59, 61, and references therein. Moreover, although this functional model can be seen as a particular case of more general results of the above papers, it proves however much more convenient for our purposes, due to the fact that it is explicitly formulated in the natural, from the point of view of the operator considered, terms. In Section 5 we characterise the absolutely continuous subspace of $A_{\varkappa}$ as the closure of the set of "smooth" vectors in the model Hilbert space introduced in Section 4. In doing so, we follow the general framework of [59, but, again, the fact that we use the specifics of a particular class of non-selfadjoint operators allows us to obtain this characterisation in a concise, easily usable form. On this basis, in Section [6] we define the wave operators for a pair from the family $\left\{A_{\varkappa}\right\}$ and demonstrate their completeness property under natural, easily verifiable assumptions. This, in combination with the functional model, allows us to obtain formulae for the scattering operator of the pair. In Section 7 we describe the representation of the scattering operator as the scattering matrix, which is explicitly written in terms of the $M$-operator.

\section{Extension theory and boundary triples}

Let $\mathcal{H}$ be a separable Hilbert space and denote by $\langle\cdot, \cdot\rangle$ the inner product in this space.

Let $A$ be a closed symmetric operator densely defined in $\mathcal{H}$, i.e. $A \subset A^{*}$, with domain $\operatorname{dom}(A) \subset$ $\mathcal{H}$. The deficiency indices $n_{+}(A), n_{-}(A)$ are defined as follows:

$$
n_{ \pm}(A):=\operatorname{dim}(\mathcal{H} \ominus \operatorname{ran}(A-z I))=\operatorname{dim}\left(\operatorname{ker}\left(A^{*}-\bar{z} I\right)\right), \quad z \in \mathbb{C}_{ \pm} .
$$

A closed operator $L$ is said to be completely non-selfadjoint if there is no subspace reducing $L$ such that the part of $L$ in this subspace is self-adjoint. A completely non-selfadjoint symmetric 
operator is often referred to as simple.

As shown in [39, Sec.1.3](see also [26, Thm.1.2.1]), the maximal invariant subspace for the closed symmetric operator $A$ in which it is self-adjoint is $\bigcap_{z \in \mathbb{C} \backslash \mathbb{R}} \operatorname{ran}(A-z I)$. Thus, a necessary and sufficient condition for the closed symmetric operator $A$ to be completely non-selfadjoint (or simple) is that

$$
\bigcap_{z \in \mathbb{C} \backslash \mathbb{R}} \operatorname{ran}(A-z I)=\{0\} .
$$

In this work we consider extensions of a given closed symmetric operator $A$ with equal deficiency indices, i. e. $n_{-}(A)=n_{+}(A)$, and use the theory of boundary triples. In order to deal with the family of extensions $\left\{A_{\varkappa}\right\}$ of the symmetric operator $A$ (where the parameter $\varkappa$ is itself an operator, see notation immediately following Proposition 2.2), we first construct a functional model of its particular dissipative extension. This is done following the Pavlov-Naboko procedure, which in turn stems from the functional model of Szökefalvi-Nagy and Foiaş. This allows us to obtain a simple model for the whole family $\left\{A_{\varkappa}\right\}$, in particular yielding a possibility to apply it to the scattering theory for certain pairs of operators in $\left\{A_{\varkappa}\right\}$, for both cases when these operators are self-adjoint and non-selfadjoint, including the possibility that both operators of the pair are non-selfadjoint.

Taking into account the importance of dissipative operators in our work, we briefly recall that a densely defined operator $L$ in $\mathcal{H}$ is called dissipative if

$$
\operatorname{Im}\langle L f, f\rangle \geq 0 \quad \forall f \in \operatorname{dom}(L) .
$$

A dissipative operator $L$ is called maximal if $\mathbb{C}_{-}$is contained in its resolvent set $\rho(L):=\{z \in \mathbb{C}$ : $\left.(L-z I)^{-1} \in \mathcal{B}(\mathcal{H})\right\} .(\mathcal{B}(\mathcal{H})$ denotes the space of bounded operators defined on the whole Hilbert space $\mathcal{H}$ ). Clearly, a maximal dissipative operator is closed; any dissipative operator admits a maximal extension.

We next describe the boundary triple approach to the extension theory of symmetric operators with equal deficiency indices (see in [16] a review of the subject). This approach has proven to be particularly useful in the study of self-adjoint extensions of ordinary differential operators of second order.

Definition 1. For a closed symmetric operator $A$ with equal deficiency indices, consider the linear mappings

$$
\Gamma_{1}: \operatorname{dom}\left(A^{*}\right) \rightarrow \mathcal{K}, \quad \Gamma_{0}: \operatorname{dom}\left(A^{*}\right) \rightarrow \mathcal{K},
$$

where $\mathcal{K}$ is an auxiliary separable Hilbert space, such that

(1) $\left\langle A^{*} f, g\right\rangle_{\mathcal{H}}-\left\langle f, A^{*} g\right\rangle_{\mathcal{H}}=\left\langle\Gamma_{1} f, \Gamma_{0} g\right\rangle_{\mathcal{K}}-\left\langle\Gamma_{0} f, \Gamma_{1} g\right\rangle_{\mathcal{K}} ;$

(2) The mapping $\operatorname{dom}\left(A^{*}\right) \ni f \mapsto\left(\begin{array}{c}\Gamma_{1} f \\ \Gamma_{0} f\end{array}\right) \in \mathcal{K} \oplus \mathcal{K}$ is surjective.

Then the triple $\left(\mathcal{K}, \Gamma_{1}, \Gamma_{0}\right)$ is said to be a boundary triple for $A^{*}$.

Remark 1. There exist boundary triples for $A^{*}$ whenever $A$ has equal deficiency indices (the case of infinite indices is not excluded), see [32, Theorem 3].

In this work we consider proper extensions of $A$, i.e. extensions of $A$ that are restrictions of $A^{*}$. The extensions $A_{B}$ for which there exists a triple $\left(\mathcal{K}, \Gamma_{1}, \Gamma_{0}\right)$ and $B \in \mathcal{B}(\mathcal{K})$ such that

$$
f \in \operatorname{dom}\left(A_{B}\right) \Longleftrightarrow \Gamma_{1} f=B \Gamma_{0} f .
$$

are called almost solvable with respect to the triple $\left(\mathcal{K}, \Gamma_{1}, \Gamma_{0}\right)$. 
Remark 2. Admittedly, the framework of almost solvable extensions is quite restrictive. In particular, even the standard three-dimensional scattering problem for PDEs in an exterior domain, with classical boundary condition (self-adjoint and non-selfadjoint alike) cannot be treated using this approach, see the discussion in [10] and also references therein. It would appear that one needs to employ the more general setting of linear relations [27, in order to accommodate this problem. However, the named setting is substantially more involved and complex than the theory of almost solvable extensions, so that the blueprints of the Sz-Nagy-Foias model of closed linear relations do not seem to be available as of today.

On the other hand, there exist at least two recent developments suggesting that the approach of the present paper can be extended beyond the natural limitations of the theory of almost solvable extensions. These are, firstly, the work [62, which offers a unified operator-theoretic approach to boundary-value problems and, in particular, an abstract definition of the $M$-operator suitable for the construction of a functional model; and secondly, the recent paper 11, which provides an explicit form of a functional model for PDE problems associated with dissipative operators. We hope to pursue this rather intriguing subject elsewhere.

The following assertions, written in slightly different terms, can be found in [32, Thm.2] and [27, Chap. 3 Sec. 1.4] (see also [60, Thm. 1.1], and [64, Sec. 14] for an alternative formulation). We compile them in the next proposition for easy reference.

Proposition 2.1. Let $A$ be a closed symmetric operator with equal deficiency indices and let $\left(\mathcal{K}, \Gamma_{1}, \Gamma_{0}\right)$ be a the boundary triple for $A^{*}$. Assume that $A_{B}$ is an almost solvable extension. Then the following statements hold:

1. $f \in \operatorname{dom}(A)$ if and only if $\Gamma_{1} f=\Gamma_{0} f=0$.

2. $A_{B}$ is maximal, i. e., $\rho\left(A_{B}\right) \neq \emptyset$.

3. $A_{B}^{*}=A_{B^{*}}$.

4. $A_{B}$ is dissipative if and only if $B$ is dissipative.

5. $A_{B}$ is self-adjoint if and only if $B$ is self-adjoint.

Definition 2. The function $M: \mathbb{C}_{-} \cup \mathbb{C}_{+} \rightarrow \mathcal{B}(\mathcal{H})$ such that

$$
M(z) \Gamma_{0} f=\Gamma_{1} f \quad \forall f \in \operatorname{ker}\left(A^{*}-z I\right)
$$

is the Weyl function of the boundary triple $\left(\mathcal{K}, \Gamma_{1}, \Gamma_{0}\right)$ for $A^{*}$, where $A$ is assumed to be as in Proposition 2.1

The Weyl function defined above has the following properties [17.

Proposition 2.2. Let $M$ be a Weyl function of the boundary triple $\left(\mathcal{K}, \Gamma_{1}, \Gamma_{0}\right)$ for $A^{*}$, where $A$ is a closed symmetric operator with equal deficiency indices. Then the following statements hold:

1. $M: \mathbb{C} \backslash \mathbb{R} \rightarrow \mathcal{B}(\mathcal{K})$.

2. $M$ is a $\mathcal{B}(\mathcal{K})$-valued double-sided $\mathcal{R}$-function [28], that is,

$$
M(z)^{*}=M(\bar{z}) \quad \text { and } \quad \operatorname{Im}(z) \operatorname{Im}(M(z))>0 \quad \text { for } z \in \mathbb{C} \backslash \mathbb{R} .
$$

3. The spectrum of $A_{B}$ coincides with the set of points $z_{0} \in \mathbb{C}$ such that $(M-B)^{-1}$ does not admit analytic continuation into $z_{0}$. 
Let us lay out the notation for some of the main objects in this paper. In the auxiliary Hilbert space $\mathcal{K}$, choose a bounded nonnegative self-adjoint operator $\alpha$ so that the operator

$$
B_{\varkappa}:=\frac{\alpha \varkappa \alpha}{2}
$$

belongs to $\mathcal{B}(\mathcal{K})$, where $\varkappa$ is a bounded operator in $E:=\operatorname{clos}(\operatorname{ran}(\alpha)) \subset \mathcal{K}$. In what follows, we deal with almost solvable extensions of a given symmetric operator $A$ that are generated by $B_{\varkappa}$ via (2.4). We always assume that the deficiency indices of $A$ are equal and that some boundary triple $\left(\mathcal{K}, \Gamma_{1}, \Gamma_{0}\right)$ for $A^{*}$ is fixed. In order to streamline the formulae, we write

$$
A_{\varkappa}:=A_{B_{\varkappa}} .
$$

Here $\varkappa$ should be understood as a parameter for a family of almost solvable extensions of $A$. Note that if $\varkappa$ is self-adjoint then so is $B_{\varkappa}$ and, hence by Proposition $2.1(5), A_{\varkappa}$ is self-adjoint. Note also that $A_{\mathrm{i} I}$ is maximal dissipative, again by Proposition 2.1

Definition 3. The characteristic function of the operator $A_{\mathrm{i} I}$ is the operator-valued function $S$ on $\mathbb{C}_{+}$given by

$$
S(z):=I \uparrow_{E}+\mathrm{i} \alpha\left(B_{\mathrm{i} I}^{*}-M(z)\right)^{-1} \alpha \uparrow_{E}, \quad z \in \mathbb{C}_{+} .
$$

In the general setting, the characteristic function is defined as in [60, Def. 1.7]. Our definition is justified by [60, Eq. 1.16].

Remark 3. The function $S$ is analytic in $\mathbb{C}_{+}$and, for each $z \in \mathbb{C}_{+}$, the mapping $S(z): E \rightarrow E$ is a contraction. Therefore, $S$ has nontangential limits almost everywhere on the real line in the strong topology [67, which we will henceforth denote by $S(k), k \in \mathbb{R}$.

Remark 4. When $\alpha=\sqrt{2} I$, an straightforward calculation yields that $S(z)$ is the Cayley transform of $M(z)$, i.e.

$$
S(z)=(M(z)-\mathrm{i} I)(M(z)+\mathrm{i} I)^{-1} .
$$

\section{Formulae for the resolvents of almost solvable extensions}

In this section we establish some useful relations between the resolvents of the operators $A_{\varkappa}$ for any $\varkappa \in \mathcal{B}(E)$ and the resolvents of the maximal dissipative operator $A_{\mathrm{i} I}$ and its adjoint. These relations ( $c f .63,59,61$ and references therein, for the corresponding results in the general setting of closed non-selfadjoint operators) are instrumental for the construction of the functional model in the next section.

Notation 1. We abbreviate

$$
\begin{array}{ll}
\Theta_{\varkappa}(z):=I-\mathrm{i} \alpha\left(B_{\mathrm{i} I}-M(z)\right)^{-1} \alpha \chi_{\varkappa}^{+}, & z \in \mathbb{C}_{-}, \\
\widehat{\Theta}_{\varkappa}(z):=I+\mathrm{i} \alpha\left(B_{\mathrm{i} I}^{*}-M(z)\right)^{-1} \alpha \chi_{\varkappa}^{-}, & z \in \mathbb{C}_{+},
\end{array}
$$

where

$$
\chi_{\varkappa}^{ \pm}:=\frac{I \pm \mathrm{i} \varkappa}{2}
$$

and for simplicity we have written $I$ instead of $I \uparrow_{E}$. We use this convention throughout the text.

It follows from Definition 3 and Proposition 2.2(2) that the operator-valued functions $\Theta_{\varkappa}(z)$ and $\widehat{\Theta}_{\varkappa}(z)$ can be expressed in terms of the characteristic function $S$, as follows:

$$
\begin{array}{ll}
\Theta_{\varkappa}(z)=I+\left(S^{*}(\bar{z})-I\right) \chi_{\varkappa}^{+} & \forall z \in \mathbb{C}_{-}, \\
\widehat{\Theta}_{\varkappa}(z)=I+(S(z)-I) \chi_{\varkappa}^{-} & \forall z \in \mathbb{C}_{+} .
\end{array}
$$


The formulae in the next lemma are analogous to [60, Eqs. 2.18 and 2.22].

Lemma 3.1. The following identities hold:

(i) $\alpha \Gamma_{0}\left(A_{\mathrm{i} I}-z I\right)^{-1}=\Theta_{\varkappa}(z) \alpha \Gamma_{0}\left(A_{\varkappa}-z I\right)^{-1} \quad \forall z \in \mathbb{C}_{-} \cap \rho\left(A_{\varkappa}\right)$;

(ii) $\alpha \Gamma_{0}\left(A_{\varkappa}-z I\right)^{-1}=\Theta_{\varkappa}(z)^{-1} \alpha \Gamma_{0}\left(A_{\mathrm{i} I}-z I\right)^{-1} \quad \forall z \in \mathbb{C}_{-} \cap \rho\left(A_{\varkappa}\right)$;

(iii) $\alpha \Gamma_{0}\left(A_{\mathrm{i} I}^{*}-z I\right)^{-1}=\widehat{\Theta}_{\varkappa}(z) \alpha \Gamma_{0}\left(A_{\varkappa}-z I\right)^{-1} \quad \forall z \in \mathbb{C}_{+} \cap \rho\left(A_{\varkappa}\right)$;

(iv) $\alpha \Gamma_{0}\left(A_{\varkappa}-z I\right)^{-1}=\widehat{\Theta}_{\varkappa}(z)^{-1} \alpha \Gamma_{0}\left(A_{\mathrm{i} I}^{*}-z I\right)^{-1} \quad \forall z \in \mathbb{C}_{+} \cap \rho\left(A_{\varkappa}\right)$.

Proof. We start by proving (ii). To this end, suppose that $z \in \mathbb{C}_{-} \cap \rho\left(A_{\varkappa}\right)$ so $\left(A_{\mathrm{i} I}-z I\right)^{-1}$ and $\left(A_{\varkappa}-z I\right)^{-1}$ are defined on the whole space $\mathcal{H}$. Fix an arbitrary $h \in \mathcal{H}$ and define

$$
\varphi:=\left(A_{\mathrm{i} I}-z I\right)^{-1} h, \quad g:=\left(A_{\varkappa}-z I\right)^{-1} h .
$$

Clearly, the vector

$$
f:=\varphi-g=\left(\left(A_{\mathrm{i} I}-z I\right)^{-1}-\left(A_{\varkappa}-z I\right)^{-1}\right) h
$$

is in $\operatorname{ker}\left(A^{*}-z I\right)$ since $A^{*}$ is an extension of both operators $A_{\mathrm{i} I}$ and $A_{\varkappa}$. According to (2.4), it follows from $\varphi \in \operatorname{dom}\left(A_{\mathrm{i} I}\right)$ and $g \in \operatorname{dom}\left(A_{\varkappa}\right)$ that $\Gamma_{1} \varphi=B_{\mathrm{i} I} \Gamma_{0} \varphi$ and $\Gamma_{1} g=B_{\varkappa} \Gamma_{0} g$. Thus, one has

$$
\begin{aligned}
0 & =\Gamma_{1}(f+g)-B_{\mathrm{i} I} \Gamma_{0}(f+g) \\
& =\Gamma_{1} f-B_{\mathrm{i} I} \Gamma_{0} f+\Gamma_{1} g-B_{\mathrm{i} I} \Gamma_{0} g \\
& =M(z) \Gamma_{0} f-B_{\mathrm{i} I} \Gamma_{0} f+B_{\varkappa} \Gamma_{0} g-B_{\mathrm{i} I} \Gamma_{0} g,
\end{aligned}
$$

where in the last equality we also use the fact that $f \in \operatorname{ker}\left(A^{*}-z I\right)$, together with Definition 2 Hence one has

$$
\Gamma_{0} f=\left(B_{\mathrm{i} I}-M(z)\right)^{-1}\left(B_{\varkappa}-B_{\mathrm{i} I}\right) \Gamma_{0} g,
$$

which, in turn, implies that

$$
\Gamma_{0} \varphi=\Gamma_{0} f+\Gamma_{0} g=\left[I+\left(B_{\mathrm{i} I}-M(z)\right)^{-1}\left(B_{\varkappa}-B_{\mathrm{i} I}\right)\right] \Gamma_{0} g .
$$

Taking into account (3.6), using the fact that $B_{\varkappa}-B_{\mathrm{i} I}=-\mathrm{i} \alpha \chi_{\varkappa}^{+} \alpha$ and applying the operator $\alpha$ to both sides of (3.7), we obtain

$$
\alpha \Gamma_{0}\left(A_{\mathrm{i} I}-z I\right)^{-1} h=\left[I-\mathrm{i} \alpha\left(B_{\mathrm{i} I}-M(z)\right)^{-1} \alpha \chi_{\varkappa}^{+}\right] \alpha \Gamma_{0}\left(A_{\varkappa}-z I\right)^{-1} h,
$$

which is the identity (i), in view of the definition (3.4).

Similar computations with the pairs $A_{\varkappa}, B_{\varkappa}$ and $A_{\mathrm{i} I}, B_{\mathrm{i} I}$ interchanged lead to

$$
\alpha \Gamma_{0}\left(A_{\varkappa}-z I\right)^{-1} h=\left[I+\mathrm{i} \alpha\left(B_{\varkappa}-M(z)\right)^{-1} \alpha \chi_{\varkappa}^{+}\right] \alpha \Gamma_{0}\left(A_{\mathrm{i} I}-z I\right)^{-1} h,
$$

for $z \in \mathbb{C}_{-} \cap \rho\left(A_{\varkappa}\right)$. Now, (iii) follows from (3.8) using the identity

$$
\Theta_{\varkappa}(z)^{-1}=I+\mathrm{i} \alpha\left(B_{\varkappa}-M(z)\right)^{-1} \alpha \chi_{\varkappa}^{+} \quad \forall z \in \mathbb{C}_{-} \cap \rho\left(A_{\varkappa}\right),
$$

which is validated by multiplying together the right-hand sides of (3.9) and (3.1) and employing a version of the second resolvent identity ( $c f$. [72, Thm. 5.13]):

$$
\left(B_{\varkappa}-M(z)\right)^{-1}-\left(B_{\mathrm{i} I}-M(z)\right)^{-1}=\left(B_{\varkappa}-M(z)\right)^{-1}\left(B_{\mathrm{i} I}-B_{\varkappa}\right)\left(B_{\mathrm{i} I}-M(z)\right)^{-1}
$$

which holds for all $z \in \mathbb{C}_{-} \cap \rho\left(A_{\varkappa}\right)$. 
We next proceed to the proof of (iii) and (iv). Fix an arbitrary $z \in \mathbb{C}_{+} \cap \rho\left(A_{\varkappa}\right)$ and an arbitrary $h \in \mathcal{H}$ and define

$$
\varphi:=\left(A_{\mathrm{i} I}^{*}-z I\right)^{-1} h, \quad g:=\left(A_{\varkappa}-z I\right)^{-1} h,
$$

then $f:=\varphi-g$ is in $\operatorname{ker}\left(A^{*}-z I\right)$. Since $\varphi \in \operatorname{dom}\left(A_{\mathrm{i} I}^{*}\right)$, one has that

$$
\begin{aligned}
0 & =\Gamma_{1}(f+g)-B_{\mathrm{i} I}^{*} \Gamma_{0}(f+g) \\
& =M(z) \Gamma_{0} f+\Gamma_{1} g-B_{\mathrm{i} I}^{*} \Gamma_{0} f-B_{\mathrm{i} I} \Gamma_{0} g,
\end{aligned}
$$

where in the second equality we use the fact that $f \in \operatorname{ker}\left(A^{*}-z I\right)$. On the other hand, in view of the inclusion $g \in \operatorname{dom}\left(A_{\varkappa}\right)$, the formula (2.4) allows us to replace the second term in the last expression by $B_{\varkappa} \Gamma_{0} g$, which yields

$$
0=\left(M(z)-B_{\mathrm{i} I}^{*}\right) \Gamma_{0} f+\left(B_{\varkappa}-B_{\mathrm{i} I}^{*}\right) \Gamma_{0} g .
$$

Since $B_{\varkappa}-B_{\mathrm{i} I}^{*}=\mathrm{i} \alpha \chi_{\varkappa}^{-} \alpha$, the equality (3.11) is rewritten as

$$
\Gamma_{0} f=\mathrm{i}\left(B_{\mathrm{i} I}^{*}-M(z)\right)^{-1} \alpha \chi_{\varkappa}^{-} \alpha \Gamma_{0} g,
$$

which in turn implies that

$$
\Gamma_{0} \varphi=\left[I+\mathrm{i}\left(B_{\mathrm{i} I}^{*}-M(z)\right)^{-1} \alpha \chi_{\varkappa}^{-} \alpha\right] \Gamma_{0} g .
$$

Applying the operator $\alpha$ to both sides of the last equation and using (3.10), we obtain

$$
\alpha \Gamma_{0}\left(A_{\mathrm{i} I}^{*}-z I\right)^{-1} h=\left[I+\mathrm{i} \alpha\left(B_{\mathrm{i} I}^{*}-M(z)\right)^{-1} \alpha \chi_{\varkappa}^{-}\right] \alpha \Gamma_{0}\left(A_{\varkappa}-z I\right)^{-1} h,
$$

which is (iii), in view of the definition (3.5).

Finally, we interchange the operators $A_{\mathrm{i} I}^{*}$ and $A_{\varkappa}$ in (3.10) and repeat the computations, correspondingly interchanging $B_{\mathrm{i} I}$ and $B_{\varkappa}$. This yields the identity

$$
\alpha \Gamma_{0}\left(A_{\varkappa}^{*}-z I\right)^{-1} h=\left[I-\mathrm{i} \alpha\left(B_{\mathrm{i} I}^{*}-M(z)\right)^{-1} \alpha \chi_{\varkappa}^{-}\right] \alpha \Gamma_{0}\left(A_{\mathrm{i} I}^{*}-z I\right)^{-1} h,
$$

for all $z \in \mathbb{C}_{+} \cap \rho\left(A_{\varkappa}\right)$. In a similar way to (3.9), we verify that

$$
\widehat{\Theta}_{\varkappa}(z)^{-1}=I-\mathrm{i} \alpha\left(B_{\mathrm{i} I}^{*}-M(z)\right)^{-1} \alpha \chi_{\varkappa}^{-} \quad \forall z \in \mathbb{C}_{+} \cap \rho\left(A_{\varkappa}\right)
$$

and hence establish (iv).

\section{Functional model and theorems about smooth vectors}

Following [48, we introduce a Hilbert space serving as a functional model for the family of operators $A_{\varkappa}$. This functional model was constructed for completely non-selfadjoint maximal dissipative operators in 53, 51, 52 and further developed in 48, 61, 59, 68. Next we recall some related necessary information. In what follows, in various formulae, we use the subscript " \pm " to indicate two different versions of the same formula in which the subscripts "+" and "-" are taken individually.

A function $f$ analytic on $\mathbb{C}_{ \pm}$and taking values in $E$ is said to be in the Hardy class $H_{ \pm}^{2}(E)$ when

$$
\sup _{y>0} \int_{\mathbb{R}}\|f(x \pm \mathrm{i} y)\|_{E}^{2} d x<+\infty
$$

(cf. [57, Sec. 4.8]). Whenever $f \in H_{ \pm}^{2}(E)$, the left-hand side of the above inequality defines $\|f\|_{H_{ \pm}^{2}(E)}^{2}$. We use the notation $H_{+}^{2}$ and $H_{-}^{2}$ for the usual Hardy spaces of $\mathbb{C}$-valued functions. 
The elements of the Hardy spaces $H_{ \pm}^{2}(E)$ are identified with their boundary values, which exist almost everywhere on the real line. We keep the same notation $H_{ \pm}^{2}(E)$ for the corresponding subspaces of $L^{2}(\mathbb{R}, E)$ [57, Sec. 4.8, Thm. B]). By the Paley-Wiener theorem [57, Sec. 4.8, Thm. E]), one verifies that these subspaces are the orthogonal complements of each other.

Following the argument of [48, Thm. 1], it is shown in [60, Lem. 2.4] that

$$
\alpha \Gamma_{0}\left(A_{\mathrm{i} I}-\cdot I\right)^{-1} h \in H_{-}^{2}(E) \quad \text { and } \quad \alpha \Gamma_{0}\left(A_{\mathrm{i} I}^{*}-\cdot I\right)^{-1} h \in H_{+}^{2}(E) .
$$

As mentioned in Remark 3 , the characteristic function $S$ given in Definition 3 has nontangential limits almost everywhere on the real line in the strong topology. Thus, for a two-component vector function $\left(\begin{array}{l}\widetilde{g} \\ g\end{array}\right)$ taking values in $E \oplus E$, one can consider the integral

$$
\int_{\mathbb{R}}\left\langle\left(\begin{array}{cc}
I & S^{*}(s) \\
S(s) & I
\end{array}\right)\left(\begin{array}{l}
\widetilde{g}(s) \\
g(s)
\end{array}\right),\left(\begin{array}{l}
\widetilde{g}(s) \\
g(s)
\end{array}\right)\right\rangle_{E \oplus E} d s,
$$

which is always nonnegative, due to the contractive properties of $S$. The space

$$
\mathfrak{H}:=L^{2}\left(E \oplus E ;\left(\begin{array}{cc}
I & S^{*} \\
S & I
\end{array}\right)\right)
$$

is the completion of the linear set of two-component vector functions $\left(\begin{array}{l}\widetilde{g} \\ g\end{array}\right): \mathbb{R} \rightarrow E \oplus E$ in the norm (4.2), factored with respect to vectors of zero norm. Naturally, not every element of the set can be identified with a pair $\left(\begin{array}{l}\widetilde{g} \\ g\end{array}\right)$ of two independent functions. Still, in what follows we keep the notation $\left(\begin{array}{l}\widetilde{g} \\ g\end{array}\right)$ for the elements of this space.

Another consequence of the contractive properties of the characteristic function $S$ is that for $\widetilde{g}, g \in L^{2}(\mathbb{R}, E)$ one has

$$
\left\|\left(\begin{array}{l}
\widetilde{g} \\
g
\end{array}\right)\right\|_{\mathfrak{H}} \geq\left\{\begin{array}{l}
\left\|\widetilde{g}+S^{*} g\right\|_{L^{2}(\mathbb{R}, E)} \\
\|S \widetilde{g}+g\|_{L^{2}(\mathbb{R}, E)}
\end{array}\right.
$$

Thus, for every Cauchy sequence $\left\{\left(\begin{array}{c}\widetilde{g}_{n} \\ g_{n}\end{array}\right)\right\}_{n=1}^{\infty}$, with respect to the $\mathfrak{H}$-topology, such that $\widetilde{g}_{n}, g_{n} \in$ $L^{2}(\mathbb{R}, E)$ for all $n \in \mathbb{N}$, the limits of $\widetilde{g}_{n}+S^{*} g_{n}$ and $S \widetilde{g}_{n}+g_{n}$ exist in $L^{2}(\mathbb{R}, E)$, so that $\widetilde{g}+S^{*} g$ and $S \widetilde{g}+g$ can always be treated as $L^{2}(\mathbb{R}, E)$ functions.

Consider the orthogonal subspaces of $\mathfrak{H}$

$$
D_{-}:=\left(\begin{array}{c}
0 \\
H_{-}^{2}(E)
\end{array}\right), \quad D_{+}:=\left(\begin{array}{c}
H_{+}^{2}(E) \\
0
\end{array}\right) .
$$

We define the space

$$
K:=\mathfrak{H} \ominus\left(D_{-} \oplus D_{+}\right),
$$

which is characterised as follows (see e.g. [51, 52]):

$$
K=\left\{\left(\begin{array}{c}
\widetilde{g} \\
g
\end{array}\right) \in \mathfrak{H}: \widetilde{g}+S^{*} g \in H_{-}^{2}(E), S \widetilde{g}+g \in H_{+}^{2}(E)\right\} .
$$

The orthogonal projection $P_{K}$ onto the subspace $K$ is given by (see e.g. [47])

$$
P_{K}\left(\begin{array}{l}
\widetilde{g} \\
g
\end{array}\right)=\left(\begin{array}{c}
\widetilde{g}-P_{+}\left(\widetilde{g}+S^{*} g\right) \\
g-P_{-}(S \widetilde{g}+g)
\end{array}\right),
$$

where $P_{ \pm}$are the orthogonal Riesz projections in $L^{2}(E)$ onto $H_{ \pm}^{2}(E)$.

A completely non-selfadjoint dissipative operator admits [67] a self-adjoint dilation. The dilation $\mathcal{A}=\mathcal{A}^{*}$ of the operator $A_{\mathrm{i} I}$ is constructed following Pavlov's procedure [51, 553, 52]: it is 
defined in the Hilbert space

$$
\mathcal{H}=L^{2}\left(\mathbb{R}_{-}, \mathcal{K}\right) \oplus \mathcal{H} \oplus L^{2}\left(\mathbb{R}_{+}, \mathcal{K}\right),
$$

so that

$$
P_{\mathcal{H}}(\mathcal{A}-z I)^{-1}\left\lceil_{\mathcal{H}}=\left(A_{\mathrm{i} I}-z I\right)^{-1}, \quad z \in \mathbb{C}_{-} .\right.
$$

As in the case of additive non-selfadjoint perturbations [48, Ryzhov established in 60, Thm. 2.3] that $\mathfrak{H}$ serves as the functional model for the dilation $\mathcal{A}$ i.e. there exists an isometry $\Phi: \mathcal{H} \rightarrow \mathfrak{H}$, which we will make explicit below in our particular setting, such that $\mathcal{A}$ is transformed into the operator of multiplication by the independent variable: $\Phi(\mathcal{A}-z I)^{-1}=(\cdot-z)^{-1} \Phi$. Furthermore, under this isometry

$$
\Phi\left\lceil_{\mathcal{H}} \mathcal{H}=K\right.
$$

unitarily, where $\mathcal{H}$ is understood as being embedded in $\mathcal{H}$ in the natural way, i.e.

$$
\mathcal{H} \ni h \mapsto 0 \oplus h \oplus 0 \in \mathcal{H}
$$

In what follows we keep the label $\Phi$ for the restriction $\Phi \curlyvee_{\mathcal{H}}$, in hope that it does not lead to confusion.

The next theorem generalises [60, Thm. 2.5], and its form is similar to [48, Thm. 3], which treats the case of additive perturbations, see also [44, 60, 59, 61, for the case of possibly non-additive perturbations. The proof blends together the arguments of [60] and [48, taking advantage of the similarity between the formulae (3.1) -(3.5) and those of [48, Section 2]. It is standard, see e.g. [44, 48, 61, and is therefore included in the Appendix for the sake of completeness only.

Theorem 4.1. (i) If $z \in \mathbb{C}_{-} \cap \rho\left(A_{\varkappa}\right)$ and $\left(\begin{array}{l}\widetilde{g} \\ g\end{array}\right) \in K$, then

$$
\Phi\left(A_{\varkappa}-z I\right)^{-1} \Phi^{*}\left(\begin{array}{l}
\widetilde{g} \\
g
\end{array}\right)=P_{K} \frac{1}{\cdot-z}\left(\begin{array}{c}
\tilde{g} \\
g-\chi_{\varkappa}^{+} \Theta_{\varkappa}^{-1}(z)\left(\widetilde{g}+S^{*} g\right)(z)
\end{array}\right) .
$$

(ii) If $z \in \mathbb{C}_{+} \cap \rho\left(A_{\varkappa}\right)$ and $\left(\begin{array}{l}\widetilde{g} \\ g\end{array}\right) \in K$, then

$$
\Phi\left(A_{\varkappa}-z I\right)^{-1} \Phi^{*}\left(\begin{array}{l}
\widetilde{g} \\
g
\end{array}\right)=P_{K} \frac{1}{\cdot-z}\left(\begin{array}{c}
\widetilde{g}-\chi_{\varkappa}^{-} \widehat{\Theta}_{\varkappa}^{-1}(z)(S \widetilde{g}+g)(z) \\
g
\end{array}\right) .
$$

Here, $\left(\widetilde{g}+S^{*} g\right)(z)$ and $(S \widetilde{g}+g)(z)$ denote the values at $z$ of the analytic continuations of the functions $\widetilde{g}+S^{*} g \in H_{-}^{2}(E)$ and $S \widetilde{g}+g \in H_{+}^{2}(E)$ into the lower half-plane and upper half-plane, respectively.

Following the ideas of Naboko, in the functional model space $\mathfrak{H}$ consider two subspaces $\mathfrak{N}_{ \pm}^{\varkappa}$ defined as follows:

$$
\mathfrak{N}_{ \pm}^{\varkappa}:=\left\{\left(\begin{array}{l}
\widetilde{g} \\
g
\end{array}\right) \in \mathfrak{H}: P_{ \pm}\left(\chi_{\varkappa}^{+}\left(\widetilde{g}+S^{*} g\right)+\chi_{\varkappa}^{-}(S \widetilde{g}+g)\right)=0\right\} .
$$

These subspaces have a characterisation in terms of the resolvent of the operator $A_{\varkappa}$. This, again, can be seen as a consequence of a much more general argument (see e.g. [61, 59]). The proof in our particular case is provided in Appendix and follows the approach of [48, Thm.4].

Theorem 4.2. Suppose that $\operatorname{ker}\{\alpha\}=0$. The following characterisation holds:

$$
\mathfrak{N}_{ \pm}^{\varkappa}=\left\{\left(\begin{array}{l}
\widetilde{g} \\
g
\end{array}\right) \in \mathfrak{H}: \Phi\left(A_{\varkappa}-z I\right)^{-1} \Phi^{*} P_{K}\left(\begin{array}{l}
\widetilde{g} \\
g
\end{array}\right)=P_{K} \frac{1}{\cdot-z}\left(\begin{array}{c}
\widetilde{g} \\
g
\end{array}\right) \text { for all } z \in \mathbb{C}_{ \pm}\right\} .
$$


Consider the counterparts of $\mathfrak{N}_{ \pm}^{\varkappa}$ in the original Hilbert space $\mathcal{H}$ :

$$
\widetilde{N}_{ \pm}^{\varkappa}:=\Phi^{*} P_{K} \mathfrak{N}_{ \pm}^{\varkappa}
$$

which are linear sets albeit not necessarily subspaces. In a way similar to [48], we introduce the set

$$
\widetilde{N}_{\mathrm{e}}^{\varkappa}:=\widetilde{N}_{+}^{\varkappa} \cap \widetilde{N}_{-}^{\varkappa}
$$

of so-called smooth vectors and its closure $N_{\mathrm{e}}^{\varkappa}:=\operatorname{clos}\left(\widetilde{N}_{\mathrm{e}}^{\varkappa}\right)$. In Section 5 we prove that $N_{\mathrm{e}}^{\varkappa}$ coincides with the absolutely continuous subspace of the operator $A_{\varkappa}$ in the case when $A_{\varkappa}=A_{\varkappa}^{*}$ and under the same additional assumption that $\operatorname{ker}(\alpha)=\{0\}$, as in Theorem 4.2 .

The next assertion ( $c f$. e.g. [61, 59], for the case of general non-selfadjoint operators), whose proof is found in Appendix, is an alternative non-model characterisation of the linear sets $\widetilde{N}_{ \pm}^{\varkappa}$.

Theorem 4.3. The sets $\widetilde{N}_{ \pm}^{\varkappa}$ are described as follows:

$$
\widetilde{N}_{ \pm}^{\varkappa}=\left\{u \in \mathcal{H}: \chi_{\varkappa}^{\mp} \alpha \Gamma_{0}\left(A_{\varkappa}-z I\right)^{-1} u \in H_{ \pm}^{2}(E)\right\} .
$$

Corollary 4.4. The right-hand side of 4 4.13) coincides with $\left\{u \in \mathcal{H}: \alpha \Gamma_{0}\left(A_{\varkappa}-z I\right)^{-1} u \in H_{ \pm}^{2}(E)\right\}$, and therefore equivalently one has

$$
\widetilde{N}_{ \pm}^{\varkappa}=\left\{u \in \mathcal{H}: \alpha \Gamma_{0}\left(A_{\varkappa}-z I\right)^{-1} u \in H_{ \pm}^{2}(E)\right\} .
$$

Proof. Indeed, if $\alpha \Gamma_{0}\left(A_{\varkappa}-z I\right)^{-1} u \in H_{+}^{2}(E)$ then clearly $\chi_{\varkappa}^{-} \alpha \Gamma_{0}\left(A_{\varkappa}-z I\right)^{-1} u \in H_{+}^{2}(E)$. Conversely, we write

$$
\begin{aligned}
S(z) \chi_{\varkappa}^{-} \alpha \Gamma_{0}\left(A_{\varkappa}-z I\right)^{-1} u & =\left(S(z) \chi_{\varkappa}^{-}+\chi_{\varkappa}^{+}\right) \alpha \Gamma_{0}\left(A_{\varkappa}-z I\right)^{-1} u-\chi_{\varkappa}^{+} \alpha \Gamma_{0}\left(A_{\varkappa}-z I\right)^{-1} u \\
& =\widehat{\Theta}_{\varkappa}(z) \alpha \Gamma_{0}\left(A_{\varkappa}-z I\right)^{-1} u-\chi_{\varkappa}^{+} \alpha \Gamma_{0}\left(A_{\varkappa}-z I\right)^{-1} u \\
& =\alpha \Gamma_{0}\left(A_{i I}^{*}-z I\right)^{-1} u-\chi_{\varkappa}^{+} \alpha \Gamma_{0}\left(A_{\varkappa}-z I\right)^{-1} u,
\end{aligned}
$$

where $S(z) \chi_{\varkappa}^{-}+\chi_{\varkappa}^{+}=(S(z)-I) \chi_{\varkappa}^{-}+I=\widehat{\Theta}_{\varkappa}(z)$, see (3.5), and in (4.16)-(4.17) we use the part (iii) of Lemma 3.1 .

Further, as we noted in (4.1), one has $\alpha \Gamma_{0}\left(A_{i I}^{*}-z I\right)^{-1} u \in H_{+}^{2}(E)$, and since $S$ is an analytic contraction in $\mathbb{C}_{+}$the function $S(z) \chi_{\varkappa}^{-} \alpha \Gamma_{0}\left(A_{\varkappa}-z I\right)^{-1} u, z \in \mathbb{C}_{+}$, is an element of $H_{+}^{2}(E)$ as long as $\chi_{\varkappa}^{-} \alpha \Gamma_{0}\left(A_{\varkappa}-z I\right)^{-1} u \in H_{+}^{2}(E)$. Recalling (4.15), (4.17), we conclude that $\chi_{\varkappa}^{+} \alpha \Gamma_{0}\left(A_{\varkappa}-z I\right)^{-1} u \in$ $H_{+}^{2}(E)$ and therefore

$$
\chi_{\varkappa}^{+} \alpha \Gamma_{0}\left(A_{\varkappa}-z I\right)^{-1} u+\chi_{\varkappa}^{-} \alpha \Gamma_{0}\left(A_{\varkappa}-z I\right)^{-1} u=\alpha \Gamma_{0}\left(A_{\varkappa}-z I\right)^{-1} u \in H_{+}^{2}(E),
$$

as required.

The equality

$$
\left\{u \in \mathcal{H}: \chi_{\varkappa}^{+} \alpha \Gamma_{0}\left(A_{\varkappa}-z I\right)^{-1} u \in H_{-}^{2}(E)\right\}=\left\{u \in \mathcal{H}: \alpha \Gamma_{0}\left(A_{\varkappa}-z I\right)^{-1} u \in H_{-}^{2}(E)\right\}
$$

is shown in a similar way.

The above corollary together with Theorem 5.5 motivates generalising the notion of the absolutely continuous subspace $\mathcal{H}_{\mathrm{ac}}\left(A_{\varkappa}\right)$ to the case of non-selfadjoint extensions $A_{\varkappa}$ of a symmetric operator $A$, by identifying it with the set $N_{\mathrm{e}}^{\varkappa}$. This generalisation follows in the footsteps of the corresponding definition by Naboko [48, in the case of additive perturbations (see also [61, 59. for the general case). In particular, an argument similar to [48, Corollary 1] shows that for the 
functional model image of $\tilde{N}_{\mathrm{e}}^{\varkappa}$ the following representation holds:

$$
\begin{aligned}
& \Phi \widetilde{N}_{\mathrm{e}}^{\varkappa}=\left\{P_{K}\left(\begin{array}{l}
\widetilde{g} \\
g
\end{array}\right) \in \mathfrak{H}:\right. \\
& \left.\left(\begin{array}{l}
\widetilde{g} \\
g
\end{array}\right) \in \mathfrak{H} \text { satisfies } \Phi\left(A_{\varkappa}-z I\right)^{-1} \Phi^{*} P_{K}\left(\begin{array}{l}
\widetilde{g} \\
g
\end{array}\right)=P_{K} \frac{1}{\cdot-z}\left(\begin{array}{c}
\widetilde{g} \\
g
\end{array}\right) \quad \forall z \in \mathbb{C}_{-} \cup \mathbb{C}_{+}\right\} .
\end{aligned}
$$

(Note that the inclusion of the right-hand side of (4.18) into $\Phi \tilde{N}_{\mathrm{e}}^{\varkappa}$ follows immediately from Theorem 4.2) Further, we arrive at an equivalent description:

$$
\Phi \widetilde{N}_{\mathrm{e}}^{\varkappa}=\left\{P_{K}\left(\begin{array}{l}
\widetilde{g} \\
g
\end{array}\right):\left(\begin{array}{l}
\widetilde{g} \\
g
\end{array}\right) \in \mathfrak{H} \text { satisfies } \chi_{\varkappa}^{+}\left(\widetilde{g}+S^{*} g\right)+\chi_{\varkappa}^{-}(S \widetilde{g}+g)=0\right\} .
$$

Definition 4. For a symmetric operator $A$, in the case of a non-selfadjoint extension $A_{\varkappa}$ the absolutely continuous subspace $\mathcal{H}_{\mathrm{ac}}\left(A_{\varkappa}\right)$ is defined by the formula $\mathcal{H}_{\mathrm{ac}}\left(A_{\varkappa}\right)=N_{\mathrm{e}}$.

In the case of a self-adjoint extension $A_{\varkappa}$, we understand $\mathcal{H}_{\mathrm{ac}}\left(A_{\varkappa}\right)$ in the sense of the classical definition of the absolutely continuous subspace of a self-adjoint operator.

\section{The relationship between the set of smooth vectors and the absolutely continuous subspace in the self-adjoint setting}

The argument of this section is similar to that of [48, subject to appropriate modifications in order to account for the fact that we deal with extensions of symmetric operators rather than additive perturbations. The same strategy seems to be applicable in the "mixed" case that incorporates both extensions and perturbations, which has recently been studied in [10].

The following proposition is contained in the proof of [48, Lemma 5]. For reader's convenience, we provide its proof in Appendix.

Proposition 5.1. If the Borel transform of a Borel measure $\mu$

$$
\int_{\mathbb{R}} \frac{d \mu(s)}{s-z}
$$

is either an element of $H_{+}^{2}$ when $z \in \mathbb{C}_{+}$or an element of $H_{-}^{2}$ when $z \in \mathbb{C}_{-}$, then $\mu$ is absolutely continuous with respect to the Lebesgue measure.

Lemma 5.2. Assume that $\varkappa=\varkappa^{*}, \operatorname{ker}(\alpha)=\{0\}$ and let $P_{S}$ be the orthogonal projection onto the singular subspace of $A_{\varkappa}$. Then following inclusion holds:

$$
P_{S} \widetilde{N}_{\mathrm{e}}^{\varkappa} \subset \bigcap_{z \in \mathbb{C} \backslash \mathbb{R}} \operatorname{ran}(A-z I) .
$$

Proof. We first demonstrate the validity of the claim for $\varkappa=0$.

We decompose each smooth vector $u$ (i.e $u \in \widetilde{N}_{\mathrm{e}}^{\varkappa}$ ) into its projections onto the absolutely continuous and singular subspaces of $A_{0}$, that is, $u=u_{\mathrm{ac}}+u_{s}$, where $u_{\mathrm{ac}} \in \mathcal{H}_{\mathrm{ac}}\left(A_{0}\right)$ and $u_{s} \in$ $\mathcal{H}_{s}\left(A_{0}\right)$, so $u_{\text {ac }} \perp u_{s}$ and $u_{s} \in P_{S} \widetilde{N}_{\mathrm{e}}^{\varkappa}$.

Consider an arbitrary $w \in \mathcal{K}$ and note that, due to the surjectivity of $\Gamma_{1}$, there exists a vector 
$v \in \operatorname{dom}\left(A^{*}\right)$ such that $\alpha w=\Gamma_{1} v$, and therefore

$$
\begin{aligned}
\left\langle\Gamma_{0}\left(A_{0}-z I\right)^{-1} u, \alpha w\right\rangle_{\mathcal{K}} & =\left\langle\Gamma_{0}\left(A_{0}-z I\right)^{-1} u, \Gamma_{1} v\right\rangle_{\mathcal{K}} \\
& =\left\langle\Gamma_{0}\left(A_{0}-z I\right)^{-1} u, \Gamma_{1} v\right\rangle_{\mathcal{K}}-\left\langle\Gamma_{1}\left(A_{0}-z I\right)^{-1} u, \Gamma_{0} v\right\rangle_{\mathcal{K}} \\
& =\left\langle\left(A_{0}-z I\right)^{-1} u, A^{*} v\right\rangle_{\mathcal{H}}-\left\langle A^{*}\left(A_{0}-z I\right)^{-1} u, v\right\rangle_{\mathcal{H}} \\
& =\int_{\mathbb{R}} \frac{1}{t-z} d \mu_{u, A^{*} v}(t)-\int_{\mathbb{R}} \frac{t}{t-z} d \mu_{u, v}(t)=\int_{\mathbb{R}} \frac{1}{t-z} d \hat{\mu}(t) .
\end{aligned}
$$

Here

$$
\mu_{u, A^{*} v}(\delta):=\left\langle E_{A_{0}}(\delta) u, A^{*} v\right\rangle_{\mathcal{H}}, \quad \mu_{u, v}(\delta):=\left\langle E_{A_{0}}(\delta) u, v\right\rangle_{\mathcal{H}} \quad \forall \text { Borel } \delta \subset \mathbb{R},
$$

where $E_{A_{0}}$ is the spectral resolution of the identity for the operator $A_{0}$, and $\hat{\mu}(t):=\mu_{u, A^{*} v}(t)-$ $t \mu_{u, v}(t)$. Furthermore, the measure $\hat{\mu}$ admits the decomposition into its absolutely continuous and singular parts with respect to the Lebesgue measure. Its singular part is equal to $\mu_{u_{s}, A^{*} v}(t)-$ $t \mu_{u_{s}, v}(t)=: \hat{\mu}_{s}(t)$, see $e . g$. 6]. The equality (5.1)-(5.2) is due to the observation that $\Gamma_{1}$ vanishes on $\operatorname{dom}\left(A_{0}\right)$, and the equality (5.2) $-(5.3)$ is a consequence of the "Green formula" (2.3) and the fact that $A \subset A_{0}$.

At the same time, it follows from Corollary 4.4 that the scalar analytic function $\left\langle\Gamma_{0}\left(A_{0}-z I\right)^{-1} u, \alpha w\right\rangle_{\mathcal{K}}$ is an element of $H_{+}^{2}$ for $z \in \mathbb{C}_{+}$and also of $H_{-}^{2}$ for $z \in \mathbb{C}_{-}$. Therefore, by Proposition 5.1 we infer from (5.1)-(5.4) that the measure $\hat{\mu}$ is absolutely continuous, which implies that its singular part $\hat{\mu}_{s}$ is the zero measure.

Finally, we invoke (5.1) -(5.4) once again, having replaced $u$ by $u_{s}$ and $\hat{\mu}$ by $\hat{\mu}_{s}$, and conclude that

$$
\left\langle\Gamma_{0}\left(A_{0}-z I\right)^{-1} u_{s}, \alpha w\right\rangle_{\mathcal{K}}=0 \quad \forall z \in \mathbb{C} \backslash \mathbb{R} .
$$

Now, by virtue of the facts that $w \in \mathcal{K}$ in (5.5) is arbitrary and $\operatorname{ker}(\alpha)=\{0\}$, it follows that $\Gamma_{0}\left(A_{0}-z I\right)^{-1} u_{s}=0$, and since $\left(A_{0}-z I\right)^{-1} u_{s} \in \operatorname{dom}\left(A_{0}\right)$ and therefore $\Gamma_{1}\left(A_{0}-z I\right)^{-1} u_{s}=0$ automatically, we obtain $\left(A_{0}-z I\right)^{-1} u_{s} \in \operatorname{dom}(A)$. Finally, since $A_{0} \supset A$, we conclude that $u_{s} \in \operatorname{ran}(A-z I)$ for all $z \in \mathbb{C} \backslash \mathbb{R}$, as claimed.

In order to treat the case of an arbitrary $\varkappa \in \mathcal{B}(\mathcal{K})$ such that $\varkappa=\varkappa^{*}$, we define "shifted" boundary operators $\widehat{\Gamma}_{0}:=\Gamma_{0}, \widehat{\Gamma}_{1}:=\Gamma_{1}-B_{\varkappa} \Gamma_{0}$. Notice that $(c f .(2.4$ (1) $)$

$$
\operatorname{dom}\left(A_{\varkappa}\right)=\left\{u \in \mathcal{H}: \Gamma_{1} u=B_{\varkappa} \Gamma_{0} u\right\}=\left\{u \in \mathcal{H}: \widehat{\Gamma}_{1} u=0\right\},
$$

i.e. the operator $A_{\varkappa}$ plays the rôle of the operator $A_{0}$ in the triple $\left(\mathcal{K}, \widehat{\Gamma}_{0}, \widehat{\Gamma}_{1}\right)$. Further, note that the change of the triple results in a change of the operator that needs to play the rôle of $A_{i I}$, the dissipative extension used to construct the functional model, which in terms of the "old" triple $\left(\mathcal{K}, \Gamma_{0}, \Gamma_{1}\right)$ should be the extension $A_{B}$ with $B=\alpha(i+\varkappa) \alpha / 2$. Repeating the above argument in this new functional model and bearing in mind that the characterisation of $\widetilde{N}_{\mathrm{e}}^{\varkappa}$ in Corollary 4.4 holds for all $\varkappa$, yields the stated result.

An immediate consequence of this result and the criterion of complete non-selfadjointness (2.1) is the following assertion.

Corollary 5.3. Let $\varkappa$ and $\alpha$ be as in the preceding lemma. If $A$ is completely non-selfadjoint, then

$$
\widetilde{N}_{\mathrm{e}}^{\varkappa} \subset \mathcal{H}_{\mathrm{ac}}\left(A_{\varkappa}\right) \text {. }
$$

We now proceed to the proof of the opposite inclusion.

Lemma 5.4 (Modified Rosenblum lemma, $c f$. [58]). Let $\beta$ be a self-adjoint operator in a Hilbert space $\mathcal{H}_{1}$. Suppose that the operator $T$, defined on $\operatorname{dom}(\beta)$ and taking values in a Hilbert space 
$\mathcal{H}_{2}$, is such that $T\left(\beta-z_{0} I\right)^{-1}$ is a Hilbert-Schmidt operator for some $z_{0} \in \rho(\beta)$. Then there exists a set $\mathcal{D}$, dense in $\mathcal{H}_{\mathrm{ac}}(\beta)$, such that

$$
\int_{\mathbb{R}}\|T \exp (-i \beta t) u\|^{2} d t<\infty
$$

for all $u \in \mathcal{D}$.

Proof. Let $x \in \mathbb{R}$ and $\epsilon>0$. By Hilbert's first identity

$$
T(\beta-(x+\mathrm{i} \epsilon) I)^{-1}=\left((x+\mathrm{i} \epsilon)-z_{0}\right) T\left(\beta-z_{0} I\right)^{-1}(\beta-(x+\mathrm{i} \epsilon) I)^{-1}+T\left(\beta-z_{0} I\right)^{-1}
$$

Consider the first term on the right-hand side of this last equation. By [49], for every $f$ in $\mathcal{H}_{1}$ the limit

$$
\lim _{\epsilon \rightarrow 0} T\left(\beta-z_{0} I\right)^{-1}(\beta-(x+\mathrm{i} \epsilon) I)^{-1} f
$$

exists for almost all $x \in \mathbb{R}$ (the convergence set actually depends on $f$ ). It follows that the limit

$$
\lim _{\epsilon \rightarrow 0} T\left((\beta-(x+\mathrm{i} \epsilon) I)^{-1}-(\beta-(x-\mathrm{i} \epsilon) I)^{-1}\right) f=: F(x)
$$

exists for all $f \in \mathcal{H}_{1}$ and almost all $x \in \mathbb{R}$.

Now, define the set

$$
\mathcal{X}(n):=\{x \in \mathbb{R}:|x|<n,\|F(x)\|<n\}
$$

If $E_{\beta}$ denotes the spectral measure of the operator $\beta$, then the set

$$
\mathcal{D}:=\bigcup_{n \in \mathbb{N}} E_{\beta}(\mathcal{X}(n)) \mathcal{H}_{\mathrm{ac}}(\beta)
$$

is dense in $\mathcal{H}_{\mathrm{ac}}(\beta)$. Consider an orthonormal basis $\left\{\phi_{k}\right\}$ in $\mathcal{H}_{2}$ and an arbitrary element $f \in \mathcal{D}$, then, for all $k$,

$$
\begin{aligned}
\left\langle T \exp (-i \beta t) f, \phi_{k}\right\rangle & =\int_{\mathcal{X}(n)} e^{-\mathrm{i} x t} \frac{d}{d x}\left\langle E_{\beta}(x) f, T^{*} \phi_{k}\right\rangle d x \\
& =\int_{\mathcal{X}(n)} e^{-\mathrm{i} x t}\left\langle F(x), T^{*} \phi_{k}\right\rangle d x,
\end{aligned}
$$

where in the last equality we have used the fact that by the spectral theorem

$$
\lim _{\epsilon \rightarrow 0}\left\langle\left((\beta-(x+\mathrm{i} \epsilon) I)^{-1}-(\beta-(x-\mathrm{i} \epsilon) I)^{-1}\right) f, \phi\right\rangle=\frac{d}{d x}\left\langle E_{\beta}(x) f, \phi\right\rangle
$$

for all $f \in \mathcal{H}_{\text {ac }}(\beta)$ and for all $\phi \in \mathcal{H}_{1}$.

By the Parseval identity one has

$$
\int_{\mathbb{R}}\left|\left\langle T \exp (-i \beta t) f, \phi_{k}\right\rangle\right|^{2} d t=2 \pi \int_{\mathcal{X}(n)}\left|\left\langle F(x), \phi_{k}\right\rangle\right|^{2} d x
$$

for all $k$, which immediately implies that

$$
\int_{\mathbb{R}}\|T \exp (-i \beta t) u\|^{2} d t=2 \pi \int_{\mathcal{X}(n)}\|F(x)\|^{2} d x \leq 4 \pi n^{3}<+\infty .
$$

Combining the above statements yields the following result. 
Theorem 5.5. Assume that $\varkappa=\varkappa^{*}, \operatorname{ker}(\alpha)=\{0\}$ and let $\alpha \Gamma_{0}\left(A_{\varkappa}-z I\right)^{-1}$ be a Hilbert-Schmidt operator for at least one point $z \in \rho\left(A_{\varkappa}\right)$. If $A$ is completely non-selfadjoint, then our definition of the absolutely continuous subspace is equivalent to the classical definition of the absolutely continuous subspace of a self-adjoint operator, i.e.

$$
N_{\mathrm{e}}^{\varkappa}=\mathcal{H}_{\mathrm{ac}}\left(A_{\varkappa}\right) .
$$

Proof. By applying the Fourier transform to the functions $\mathbb{1}_{ \pm}(t) \alpha \Gamma_{0} e^{i A_{\varkappa} t} e^{\mp \epsilon t} u, t \in \mathbb{R}$, where $\mathbb{1}_{ \pm}$ is the characteristic function of $\mathbb{R}_{ \pm}$and $\epsilon>0$ is arbitrarily small, one obtains

$$
\left\|\alpha \Gamma_{0}\left(A_{\varkappa}-z I\right)^{-1} u\right\|_{H_{-}^{2}}^{2}+\left\|\alpha \Gamma_{0}\left(A_{\varkappa}-z I\right)^{-1} u\right\|_{H_{+}^{2}}^{2}=2 \pi \int_{\mathbb{R}}\left\|\alpha \Gamma_{0} \exp \left(i A_{\varkappa} t\right) u\right\|^{2} d t
$$

which by Lemma 5.4 is finite for all $u$ in a dense subset of $\mathcal{H}_{\text {ac }}\left(A_{\varkappa}\right)$. Hence, in view of Corollary 4.4 and performing closure, one has $\mathcal{H}_{\mathrm{ac}}\left(A_{\varkappa}\right) \subset N_{\mathrm{e}}^{\varkappa}$. Taking into account Corollary 5.3 completes the proof.

Remark 5. Alternative conditions, which are less restrictive in general, that guarantee the validity of the assertion of Theorem 5.5 can be obtained along the lines of [50].

\section{Wave and scattering operators}

The results of the preceding sections allow us to calculate the wave operators for any pair $A_{\varkappa_{1}}, A_{\varkappa_{2}}$, where $A_{\varkappa_{1}}$ and $A_{\varkappa_{2}}$ are operators in the class introduced in Section 2, under the additional assumption that the operator $\alpha$ (see (2.5)) has a trivial kernel. For simplicity, in what follows we set $\varkappa_{2}=0$ and write $\varkappa$ instead of $\varkappa_{1}$. Note that $A_{0}$ is a self-adjoint operator, which is convenient for presentation purposes.

We begin by establishing the model representation for the function $\exp \left(i A_{\varkappa} t\right), t \in \mathbb{R}$, of the operator $A_{\varkappa}$, evaluated on the set of smooth vectors $\widetilde{N}_{\mathrm{e}}^{\varkappa}$.

Proposition 6.1. ([48, Prop. 2]) For all $t \in \mathbb{R}$ and all $\left(\begin{array}{l}\widetilde{g} \\ g\end{array}\right)$ such that $\Phi^{*} P_{K}\left(\begin{array}{l}\widetilde{g} \\ g\end{array}\right) \in \widetilde{N}_{\mathrm{e}}^{\varkappa}$ one has

$$
\Phi \exp \left(i A_{\varkappa} t\right) \Phi^{*} P_{K}\left(\begin{array}{l}
\widetilde{g} \\
g
\end{array}\right)=P_{K} \exp (i k t)\left(\begin{array}{l}
\widetilde{g} \\
g
\end{array}\right) .
$$

Proof. We use the definition

$$
\exp \left(i A_{\varkappa} t\right):=\operatorname{sim}_{n \rightarrow+\infty}\left(I-\frac{i A_{\varkappa} t}{n}\right)^{-n}, \quad t \in \mathbb{R}
$$

giving in general an unbounded operator (see [29]). Due to Theorem 4.2, if $\left(\begin{array}{l}\widetilde{g} \\ g\end{array}\right) \in \mathfrak{N}_{+}^{\varkappa} \cap \mathfrak{N}_{-}^{\varkappa}$, i.e. $\Phi^{*} P_{K}\left(\begin{array}{c}\widetilde{g} \\ g\end{array}\right) \in \widetilde{N}_{\mathrm{e}}^{\varkappa}$, then

$$
\left(I-\frac{i A_{\varkappa} t}{n}\right)^{-n} \Phi^{*} P_{K}\left(\begin{array}{l}
\widetilde{g} \\
g
\end{array}\right)=\Phi^{*} P_{K}\left(1-\frac{i k t}{n}\right)^{-n}\left(\begin{array}{l}
\widetilde{g} \\
g
\end{array}\right), \quad t \in \mathbb{R} .
$$

Thus, to complete the proof it remains to show that

$$
\left\|\left(\exp (i k t)-\left(1-\frac{i k t}{n}\right)^{-n}\right)\left(\begin{array}{l}
\widetilde{g} \\
g
\end{array}\right)\right\|_{\mathfrak{H}} \underset{n \rightarrow \infty}{\longrightarrow} 0, \quad t \in \mathbb{R},
$$

which follows directly from Lebesgue's dominated convergence theorem. 
Proposition 6.2. (㥜, Section 4]) If $\Phi^{*} P_{K}\left(\begin{array}{c}\widetilde{g} \\ g\end{array}\right) \in \widetilde{N}_{\mathrm{e}}^{\varkappa}$ and $\Phi^{*} P_{K}\left(\begin{array}{l}\widehat{g} \\ g\end{array}\right) \in \widetilde{N}_{\mathrm{e}}^{0}$ (with the same elemen 1 g), then

$$
\left\|\exp \left(-i A_{\varkappa} t\right) \Phi^{*} P_{K}\left(\begin{array}{c}
\widetilde{g} \\
g
\end{array}\right)-\exp \left(-i A_{0} t\right) \Phi^{*} P_{K}\left(\begin{array}{c}
\widehat{g} \\
g
\end{array}\right)\right\|_{\mathfrak{H}} \underset{t \rightarrow-\infty}{\longrightarrow} 0 .
$$

Proof. Clearly, $\widetilde{g}-\widehat{g} \in L^{2}(E)$ since $\left(\begin{array}{c}\widetilde{g}-\widehat{g} \\ 0\end{array}\right) \in \mathfrak{H}$. Therefore, for all $t \in \mathbb{R}$, we obtain

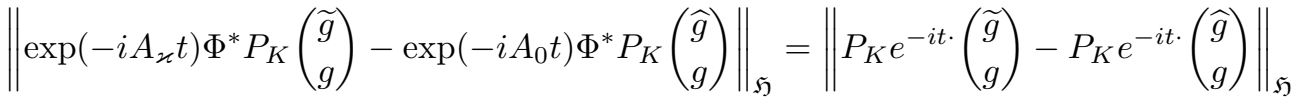

$$
\begin{aligned}
& =\left\|P_{K}\left(\begin{array}{c}
e^{-i t \cdot}(\widetilde{g}-\widehat{g}) \\
0
\end{array}\right)\right\|_{\mathfrak{H}} \\
& \leq\left\|P_{-} e^{-i t \cdot}(\widetilde{g}-\widehat{g})\right\|_{L^{2}(E)} \text {. }
\end{aligned}
$$

where in the inequality we use the fact that

$$
\left\|P_{K}\left(\begin{array}{c}
\check{g} \\
0
\end{array}\right)\right\|_{\mathfrak{H}}^{2}=\int_{\mathbb{R}}\left(\left\|P_{-} \check{g}(s)\right\|_{E}^{2}-\left\|P_{-} S(s) \check{g}(s)\right\|_{E}^{2}\right) d s \quad \forall\left(\begin{array}{c}
\check{g} \\
0
\end{array}\right) \in \mathfrak{H} .
$$

Finally, since $\exp (-i t \cdot) \in H_{+}^{\infty}$ for $t \geq 0$, the convergence (see e.g. [34])

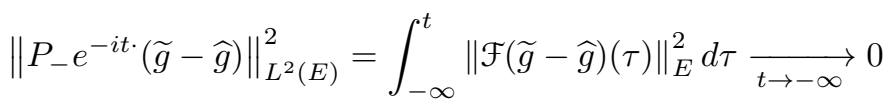

holds, where $\mathcal{F}(\widetilde{g}-\widehat{g})$ stands for the Fourier transform of the function $\widetilde{g}-\widehat{g}$.

It follows from Proposition 6.2 that whenever $\Phi^{*} P_{K}\left(\begin{array}{c}\widetilde{g} \\ g\end{array}\right) \in \widetilde{N}_{\mathrm{e}}^{\varkappa}$ and $\Phi^{*} P_{K}\left(\begin{array}{l}\widehat{g} \\ g\end{array}\right) \in \widetilde{N}_{\mathrm{e}}^{0}$ (with the same second component $g$ ), formally one has

$$
\begin{aligned}
\lim _{t \rightarrow-\infty} e^{i A_{0} t} e^{-i A_{\varkappa} t} \Phi^{*} P_{K}\left(\begin{array}{l}
\widetilde{g} \\
g
\end{array}\right) & =\Phi^{*} P_{K}\left(\begin{array}{l}
\widehat{g} \\
g
\end{array}\right) \\
& =\Phi^{*} P_{K}\left(\begin{array}{c}
-(I+S)^{-1}\left(I+S^{*}\right) g \\
g
\end{array}\right),
\end{aligned}
$$

where in the last equality we use the inclusion $\Phi^{*} P_{K}\left(\begin{array}{l}\widehat{g} \\ g\end{array}\right) \in \widetilde{N}_{\mathrm{e}}^{0}$, which by (4.19) yields $\widehat{g}+S^{*} g+$ $S \widehat{g}+g=0$. In view of the classical definition of the wave operator of a pair of self-adjoint operators, see e.g. [29],

$$
W_{ \pm}\left(A_{0}, A_{\varkappa}\right):=\underset{t \rightarrow \pm \infty}{\mathrm{s}-\lim } e^{i A_{0} t} e^{-i A_{\varkappa} t} P_{\mathrm{ac}}^{\varkappa}
$$

where $P_{\mathrm{ac}}^{\varkappa}$ is the projection onto the absolutely continuous subspace of $A^{\varkappa}$, we obtain that, at least formally, for $\Phi^{*} P_{K}\left(\begin{array}{c}\widetilde{g} \\ g\end{array}\right) \in \widetilde{N}_{\mathrm{e}}^{\varkappa}$ one has

$$
W_{-}\left(A_{0}, A_{\varkappa}\right) \Phi^{*} P_{K}\left(\begin{array}{c}
\widetilde{g} \\
g
\end{array}\right)=\Phi^{*} P_{K}\left(\begin{array}{c}
-(I+S)^{-1}\left(I+S^{*}\right) g \\
g
\end{array}\right) .
$$

By an argument similar to that of Proposition 6.2 (i.e. considering the case $t \rightarrow+\infty$ ), one also

\footnotetext{
${ }^{1}$ Despite the fact that $\left(\begin{array}{l}\widetilde{g} \\ g\end{array}\right) \in \mathfrak{H}$ is nothing but a symbol, still $\widetilde{g}$ and $g$ can be identified with vectors in certain $L^{2}(E)$ spaces with operators "weights", see details below in Section 7 Further, we recall that even then for $\left(\begin{array}{l}\widetilde{g} \\ g\end{array}\right) \in \mathfrak{H}$, the components $\widetilde{g}$ and $g$ are not, in general, independent of each other.
} 
obtains

$$
W_{+}\left(A_{0}, A_{\varkappa}\right) \Phi^{*} P_{K}\left(\begin{array}{c}
\widetilde{g} \\
g
\end{array}\right)=\lim _{t \rightarrow+\infty} e^{i A_{0} t} e^{-i A_{\varkappa} t} \Phi^{*} P_{K}\left(\begin{array}{c}
\widetilde{g} \\
g
\end{array}\right)=\Phi^{*} P_{K}\left(\begin{array}{c}
\widetilde{g} \\
-\left(I+S^{*}\right)^{-1}(I+S) \widetilde{g}
\end{array}\right)
$$

again for $\Phi^{*} P_{K}\left(\begin{array}{c}\widetilde{g} \\ g\end{array}\right) \in \widetilde{N}_{\mathrm{e}}^{\varkappa}$.

Further, the definition of the wave operators $W_{ \pm}\left(A_{\varkappa}, A_{0}\right)$

$$
\left\|e^{-i A_{\varkappa} t} W_{ \pm}\left(A_{\varkappa}, A_{0}\right) \Phi^{*} P_{K}\left(\begin{array}{c}
\widetilde{g} \\
g
\end{array}\right)-e^{-i A_{0} t} \Phi^{*} P_{K}\left(\begin{array}{c}
\widetilde{g} \\
g
\end{array}\right)\right\|_{\mathfrak{H}} \underset{t \rightarrow \pm \infty}{\longrightarrow} 0
$$

yields, for all $\Phi^{*} P_{K}\left(\begin{array}{c}\widetilde{g} \\ g\end{array}\right) \in \widetilde{N}_{\mathrm{e}}^{0}$

$$
W_{-}\left(A_{\varkappa}, A_{0}\right) \Phi^{*} P_{K}\left(\begin{array}{l}
\widetilde{g} \\
g
\end{array}\right)=\Phi^{*} P_{K}\left(\begin{array}{c}
-\left(I+\chi_{\varkappa}^{-}(S-I)\right)^{-1}\left(I+\chi_{\varkappa}^{+}\left(S^{*}-I\right)\right) g \\
g
\end{array}\right)
$$

and

$$
W_{+}\left(A_{\varkappa}, A_{0}\right) \Phi^{*} P_{K}\left(\begin{array}{l}
\widetilde{g} \\
g
\end{array}\right)=\Phi^{*} P_{K}\left(\begin{array}{c}
\widetilde{g} \\
-\left(I+\chi_{\varkappa}^{+}\left(S^{*}-I\right)\right)^{-1}\left(I+\chi_{\varkappa}^{-}(S-I)\right) \widetilde{g}
\end{array}\right),
$$

where we have used the fact that $\Phi^{*} P_{K}\left(\begin{array}{c}\widetilde{g} \\ g\end{array}\right) \in \widetilde{N}_{\mathrm{e}}^{\varkappa}$ and the corresponding criterion provided by (4.19).

In order to rigorously justify the above formal argument, i.e. in order to prove the existence and completeness of the wave operators, one needs to first show that the right-hand sides of the formulae (6.1) - 6.4 make sense on dense subsets of the corresponding absolutely continuous subspaces. Noting that (6.1) - (6.4) have the form identical to the expressions for wave operators derived in [48, Section 4], [50, the remaining part of this justification is a modification of the argument of [50], as follows.

Let $S(z)-I$ be of the class $\mathfrak{S}_{\infty}\left(\overline{\mathbb{C}}_{+}\right)$, i.e. a compact analytic operator function in the upper half-plane up to the real line. Then so is $(S(z)-I) / 2$, which is also uniformly bounded in the upper half-plane along with $S(z)$. We next use the result of [50, Theorem 3] about the non-tangential boundedness of operators of the form $(I+T(z))^{-1}$ for $T(z)$ compact up to the real line. We infer that, provided $\left(I+\left(S\left(z_{0}\right)-I\right) / 2\right)^{-1}$ exists for some $z_{0} \in \mathbb{C}_{+}$(and hence, see [9], everywhere in $\mathbb{C}_{+}$except for a countable set of points accumulating only to the real line), one has non-tangential boundedness of $(I+(S(z)-I) / 2)^{-1}$, and therefore also of $(I+S(z))^{-1}$, for almost all points of the real line.

On the other hand, the latter inverse can be computed in $\mathbb{C}_{+}$:

$$
(I+S(z))^{-1}=\frac{1}{2}\left(I+i \alpha M(z)^{-1} \alpha / 2\right) .
$$

Indeed, one has

$$
\begin{aligned}
& \left(I+i \alpha M(z)^{-1} \alpha / 2\right)(I+S(z)) \\
& \quad=2 I+i \alpha M(z)^{-1} \alpha+i \alpha\left(B_{i I}^{*}-M(z)\right)^{-1} \alpha-i \alpha M(z)^{-1} B_{i I}^{*}\left(B_{i I}^{*}-M(z)\right)^{-1} \alpha=2 I
\end{aligned}
$$

and the second similar identity for the multiplication in the reverse order proves the claim.

It follows from (6.5) and the analytic properties of $M(z)$ that the inverse $(I+S(z))^{-1}$ exists everywhere in the upper half-plane. Thus, Theorem 3 of [50] is indeed applicable, which yields that $(I+S(z))^{-1}$ is $\mathbb{R}$-a.e. nontangentially bounded and, by the operator generalisation of the Calderon theorem (see [65]), which was extended to the operator context in [50, Theorem 1], it admits measurable non-tangential limits in the strong operator topology almost everywhere on $\mathbb{R}$. As it is easily seen, these limits must then coincide with $(I+S(k))^{-1}$ for almost all $k \in \mathbb{R}$. 
The same argument obviously applies to $\left(I+S^{*}(\bar{z})\right)^{-1}$ for $z \in \mathbb{C}_{-}$, where the invertibility follows from the identity

$$
\left(I+S^{*}(\bar{z})\right)^{-1}=\frac{1}{2}\left(I-i \alpha M(z)^{-1} \alpha / 2\right)
$$

obtained exactly as (6.5), by taking into account analytic properties of $M(z)$.

Finally, the identities

$$
\left(I+\chi_{\varkappa}^{-}(S(z)-I)\right)^{-1}=I-i \chi_{\varkappa}^{-} \alpha\left(B_{\varkappa}-M(z)\right)^{-1} \alpha
$$

for $z \in \mathbb{C}_{+}$and

$$
\left(I+\chi_{\varkappa}^{+}\left(S^{*}(\bar{z})-I\right)\right)^{-1}=I+i \chi_{\varkappa}^{+} \alpha\left(B_{\varkappa}-M(z)\right)^{-1} \alpha
$$

for $z \in \mathbb{C}_{-}$are used, again by an application of Theorem 3 of [50], to ascertain the existence of bounded $\left(I+\chi_{\varkappa}^{-}(S(k)-I)\right)^{-1}$ and $\left(I+\chi_{\varkappa}^{+}\left(S^{*}(k)-I\right)\right)^{-1}$ almost everywhere on $\mathbb{R}$, provided that the operator $A_{\varkappa}$ has at least one regular point in each half-plane of the complex plane, see Proposition 2.2. Under the assumptions on $S$ specified above, this latter condition immediately implies that the non-real spectrum of $A_{\varkappa}$ is countable and accumulates to $\mathbb{R}$ only. (Nevertheless, it could still accumulate to all points of the real line simultaneously.)

The presented argument allows one to verify the correctness of the formulae (6.1)-(6.4) for the wave operators. Indeed, for the first of them one considers $\mathbb{1}_{n}(k)$, the indicator of the set $\left\{k \in \mathbb{R}:\left\|(I+S(k))^{-1}\right\| \leq n\right\}$. Clearly, $\mathbb{1}_{n}(k) \rightarrow 1$ as $n \rightarrow \infty$ for almost all $k \in \mathbb{R}$. Next, suppose that $P_{K}(\tilde{g}, g) \in \tilde{N}_{\mathrm{e}}^{\varkappa}$. Then $P_{K} \mathbb{1}_{n}(\tilde{g}, g)$ is also a smooth vector and

$$
\left(\begin{array}{c}
-(I+S)^{-1} \mathbb{1}_{n}\left(I+S^{*}\right) g \\
\mathbb{1}_{n} g
\end{array}\right) \in \mathfrak{H} .
$$

Indeed, for any $(\tilde{g}, g) \in \mathfrak{H}$ one has

$$
\begin{aligned}
\left(\begin{array}{c}
-\mathbb{1}_{n}(1+S)^{-1}\left(1+S^{*}\right) g \\
\mathbb{1}_{n} g
\end{array}\right)-\left(\begin{array}{c}
\mathbb{1}_{n} \tilde{g} \\
\mathbb{1}_{n} g
\end{array}\right) & \\
& =\left(\begin{array}{c}
-\mathbb{1}_{n}(1+S)^{-1}\left[\left(\tilde{g}+S^{*} g\right)+(S \tilde{g}+g)\right] \\
0
\end{array}\right) \in\left(\begin{array}{c}
L^{2}(E) \\
0
\end{array}\right) \in \mathfrak{H},
\end{aligned}
$$

whereas the inclusion in the set of smooth vectors follows directly from (4.19). It follows, by the Lebesgue dominated convergence theorem, that the set of vectors $P_{K} \mathbb{1}_{n}(\tilde{g}, g)$ is dense in $N_{\mathrm{e}}^{\varkappa}$. The remaining three wave operators are treated in a similar way. Finally, the density of the range of the four wave operators follows from the density of their domains, by a standard inversion argument, see e.g. [73].

We have thus proved the following theorem.

Theorem 6.3. Let $A$ be a closed, symmetric, completely nonselfadjoint operator with equal deficiency indices and consider its extension $A_{\varkappa}$, as described in Section Q , under the assumptions that $\operatorname{ker}(\alpha)=\{0\}$ (see (2.5)) and that $A_{\varkappa}$ has at least one regular point in $\mathbb{C}_{+}$and in $\mathbb{C}_{-}$. If $S-I \in \mathfrak{S}_{\infty}\left(\overline{\mathbb{C}}_{+}\right)$, then the wave operators $W_{ \pm}\left(A_{0}, A_{\varkappa}\right)$ and $W_{ \pm}\left(A_{\varkappa}, A_{0}\right)$ exist on dense sets in $N_{\mathrm{e}}$ and $\mathcal{H}_{\mathrm{ac}}\left(A_{0}\right)$, respectively, and are given by the formulae (6.1)-6.4). The ranges of $W_{ \pm}\left(A_{0}, A_{\varkappa}\right)$ and $W_{ \pm}\left(A_{\varkappa}, A_{0}\right)$ are dense in $\mathcal{H}_{\mathrm{ac}}\left(A_{0}\right)$ and $N_{\mathrm{e}}^{\varkappa}$, respectively $\mathrm{L}^{2}$

Remark 6. 1 . The identities (6.5)-(6.6) can be used to replace the condition $S(z)-I \in \mathfrak{S}_{\infty}\left(\overline{\mathbb{C}}_{+}\right)$ by the following equivalent condition: $\alpha M(z)^{-1} \alpha$ is nontangentially bounded almost everywhere

\footnotetext{
${ }^{2}$ In the case when $A_{\varkappa}$ is self-adjoint, or, in general, the named wave operators are bounded, the claims of the theorem are equivalent (by the classical Banach-Steinhaus theorem) to the statement of the existence and completeness of the wave operators for the pair $A_{0}, A_{\varkappa}$. Sufficient conditions of boundedness of these wave operators are contained in e.g. [48, Section 4], [50] and references therein.
} 
on the real line, and $\alpha M(z)^{-1} \alpha \in \mathfrak{S}_{\infty}\left(\overline{\mathbb{C}}_{+}\right)$for $\Im z \geq 0$. In order to do so, one notes that $(I+T)^{-1}-I=-(I+T)^{-1} T \in \mathfrak{S}_{\infty}\left(\overline{\mathbb{C}}_{+}\right)$as long as $T \in \mathfrak{S}_{\infty}\left(\overline{\mathbb{C}}_{+}\right)$and $(I+T)^{-1}$ is bounded.

2. The latter condition is satisfied [24], as long as the scalar function $\left\|\alpha M(z)^{-1} \alpha\right\|_{\mathfrak{S}_{p}}$ is nontangentially bounded almost everywhere on the real line for some $p<\infty$, where $\mathfrak{S}_{p}, p \in(0, \infty]$, are the standard Schatten - von Neumann classes of compact operators.

3. An alternative sufficient condition is the condition $\alpha \in \mathfrak{S}_{2}$ (and therefore $B_{\varkappa} \in \mathfrak{S}_{1}$ ), or, more generally, $\alpha M(z)^{-1} \alpha \in \mathfrak{S}_{1}$, see [4] for details.

4. Following from the analysis above, the existence and completeness of the wave operators for the par $A_{\varkappa}, A_{0}$ is closely linked to the condition of $\alpha$ having a "relative Hilbert-Schmidt property" with respect to $M(z)$. Recalling that $B_{\varkappa}=\alpha \varkappa \alpha / 2$, this is not always feasible to expect. Nevertheless, by appropriately modifying the boundary triple, the situation can often be rectified. For example, if $C_{\varkappa}=C_{0}+\alpha \varkappa \alpha / 2$, where $C_{0}$ and $\varkappa$ are bounded and $\alpha \in \mathfrak{S}_{2}$, replaces the operator $B_{\varkappa}$ in (2.5), then one "shifts" the boundary triple ( $c f$. the proof of Lemma [5.2): $\widehat{\Gamma}_{0}=\Gamma_{0}$, $\widehat{\Gamma}_{1}=\Gamma_{1}-C_{0} \Gamma_{0}$. One thus obtains that in the new triple $\left(\mathcal{K}, \widehat{\Gamma}_{0}, \widehat{\Gamma}_{1}\right)$ the operator $A_{\varkappa}$ coincides with the extension corresponding to the boundary operator $B_{\varkappa}=\alpha \varkappa \alpha / 2$, whereas the Weyl-Titchmarsh function $M(z)$ undergoes a shift to the function $M(z)-C_{0}$. The proof of Theorem 6.1 remains intact, while Part 3 of this remark yields that the condition $\alpha\left(M(z)-C_{0}\right)^{-1} \alpha \in \mathfrak{S}_{1}$ guarantees the existence and completeness of the wave operators for the pair $A_{C_{0}}, A_{C_{\varkappa}}$. The fact that the operator $A_{0}$ here is replaced by the operator $A_{C_{0}}$ reflects the standard argument that the complete scattering theory for a pair of operators requires that the operators forming this pair are "close enough" to each other.

Finally, the scattering operator $\Sigma$ for the pair $A_{\varkappa}, A_{0}$ is defined by

$$
\Sigma=W_{+}^{-1}\left(A_{\varkappa}, A_{0}\right) W_{-}\left(A_{\varkappa}, A_{0}\right) .
$$

The above formulae for the wave operators lead ( $c f$. [48]) to the following formula for the action of $\Sigma$ in the model representation:

$$
\Phi \Sigma \Phi^{*} P_{K}\left(\begin{array}{c}
\tilde{g} \\
g
\end{array}\right)=P_{K}\left(\begin{array}{c}
-\left(I+\chi_{\varkappa}^{-}(S-I)\right)^{-1}\left(I+\chi_{\varkappa}^{+}\left(S^{*}-I\right)\right) g \\
\left(I+S^{*}\right)^{-1}(I+S)\left(I+\chi_{\varkappa}^{-}(S-I)\right)^{-1}\left(I+\chi_{\varkappa}^{+}\left(S^{*}-I\right)\right) g
\end{array}\right),
$$

whenever $\Phi^{*} P_{K}\left(\begin{array}{l}\tilde{g} \\ g\end{array}\right) \in \widetilde{N}_{\mathrm{e}}^{0}$. In fact, as explained above, this representation holds on a dense linear set in $\tilde{N}_{\mathrm{e}}^{0}$ within the conditions of Theorem 6.3. which guarantees that all the objects on the right-hand side of the formula (6.9) are correctly defined.

\section{Spectral representation for the absolutely continuous part of the operator $A_{0}$}

The identity

$$
\left\|P_{K}\left(\begin{array}{l}
\tilde{g} \\
g
\end{array}\right)\right\|_{\mathfrak{H}}^{2}=\left\langle\left(I-S^{*} S\right) \tilde{g}, \tilde{g}\right\rangle
$$

which is derived in the same way as in [48, Section 7] for all $P_{K}\left(\begin{array}{l}\tilde{g} \\ g\end{array}\right) \in \widetilde{N}_{\mathrm{e}}^{0}$ and is equivalent to the condition $\left(\tilde{g}+S^{*} g\right)+(S \tilde{g}+g)=0$, see (4.19), allows us to consider the isometry $F: \Phi \widetilde{N}_{\mathrm{e}}^{0} \mapsto$ $L^{2}\left(E ; I-S^{*} S\right)$ defined by the formula

$$
F P_{K}\left(\begin{array}{l}
\tilde{g} \\
g
\end{array}\right)=\tilde{g} .
$$


Here $L^{2}\left(E ; I-S^{*} S\right)$ is the Hilbert space of $E$-valued functions on $\mathbb{R}$ square summable with the matrix "weight" $I-S^{*} S$, cf. (4.3). Similarly, the formula

$$
F_{*} P_{K}\left(\begin{array}{c}
\tilde{g} \\
g
\end{array}\right)=g
$$

defines an isometry $F_{*}$ from $\Phi \widetilde{N}_{\mathrm{e}}^{0}$ to $L^{2}\left(E ; I-S S^{*}\right)$.

Lemma 7.1. Suppose that the assumptions of Theorem 6.3 hold. Then the ranges of the operators $F$ and $F_{*}$ are dense in the spaces $L^{2}\left(E ; I-S^{*} S\right)$ and $L^{2}\left(E ; I-S S^{*}\right)$, respectively.

Proof. Indeed, for all $\tilde{g} \in L^{2}\left(E ; I-S^{*} S\right)$ and $g=-S \tilde{g}$ one has $(\tilde{g}, g) \in \mathcal{H}$ with $\|(\tilde{g}, g)\|_{\mathcal{H}}=$ $\|\tilde{g}\|_{L^{2}\left(E ; I-S^{*} S\right)}$. By repeating the proof of Theorem 6.3 the operator $I+S^{*}$ is boundedly invertible almost everywhere on $\mathbb{R}$.

Further, consider $\mathbb{1}_{n}(k)$, the indicator of the set $\left\{k \in \mathbb{R}:\left\|\left(I+S^{*}(k)\right)^{-1}\right\| \leq n\right\}$. For $\tilde{g} \in$ $L^{2}\left(E ; I-S^{*} S\right)$ and, as above, $g=-S \tilde{g}$, one has $\mathbb{1}_{n}\left(\tilde{g},-\left(I+S^{*}\right)^{-1}(I+S) \tilde{g}\right) \in \mathcal{H}$, since

$$
\begin{aligned}
\mathbb{1}_{n}\left(\begin{array}{c}
\tilde{g} \\
-\left(I+S^{*}\right)^{-1}(I+S) \tilde{g}
\end{array}\right)-\mathbb{1}_{n}\left(\begin{array}{l}
\tilde{g} \\
g
\end{array}\right) & \\
= & \left(\begin{array}{c}
0 \\
-\mathbb{1}_{n}\left(I+S^{*}\right)^{-1}\left[(S \tilde{g}+g)+\left(\tilde{g}+S^{*} g\right)\right]
\end{array}\right) \in\left(\begin{array}{c}
0 \\
L^{2}(E)
\end{array}\right) .
\end{aligned}
$$

Finally, the set $\left\{\mathbb{1}_{n} \tilde{g}\right\}$ is dense in $L^{2}\left(E ; I-S^{*} S\right)$ by the Lebesgue dominated convergence theorem, whereas $P_{K} \mathbb{1}_{n}\left(\tilde{g},-\left(I+S^{*}\right)^{-1}(I+S) \tilde{g}\right) \in \widetilde{N}_{\mathrm{e}}^{0}$ by direct calculation.

Corollary 7.2. The operator $F$, respectively $F_{*}$, admits an extension to the unitary mapping between $\Phi N_{\mathrm{e}}^{0}$ and $L^{2}\left(E ; I-S^{*} S\right)$, respectively $L^{2}\left(E ; I-S S^{*}\right)$.

It follows that the operator $\left(A_{0}-z\right)^{-1}$ (see notation (2.6) ) considered on $\widetilde{N}_{\mathrm{e}}^{0}$ acts as the multiplication by $(k-z)^{-1}, k \in \mathbb{R}$, both in $L^{2}\left(E ; I-S^{*} S\right)$ and $L^{2}\left(E ; I-S S^{*}\right)$. In particular, if one considers the absolutely continuous "part" of the operator $A_{0}$, namely the operator $A_{0}^{(\mathrm{e})}:=$ $\left.A_{0}\right|_{N_{\mathrm{e}}^{0}}$, then $F \Phi A_{0}^{(\mathrm{e})} \Phi^{*} F^{*}$ and $F_{*} \Phi A_{0}^{(\mathrm{e})} \Phi^{*} F_{*}^{*}$ are the operators of multiplication by the independent variable in the spaces $L^{2}\left(E ; I-S^{*} S\right)$ and $L^{2}\left(E ; I-S S^{*}\right)$, respectively.

In order to obtain a spectral representation from the above result, it is necessary to diagonalise the weights in the definitions of the above $L^{2}$-spaces. The corresponding transformation is straightforward when $\alpha=\sqrt{2} I$. (This choice of $\alpha$ satisfies the conditions of Theorem 6.3 e.g. when the boundary space $\mathcal{K}$ is finite-dimensional. The corresponding diagonalisation in the general setting of non-negative, bounded $\alpha$ will be treated elsewhere.) In this particular case one has

$$
S=(M-i I)(M+i I)^{-1},
$$

and consequently

$$
I-S^{*} S=-2 i\left(M^{*}-i I\right)^{-1}\left(M-M^{*}\right)(M+i I)^{-1}
$$

and

$$
I-S S^{*}=2 i(M+i I)^{-1}\left(M^{*}-M\right)\left(M^{*}-i I\right)^{-1} .
$$

Introducing the unitary transformations

$$
\begin{aligned}
G: L^{2}\left(E ; I-S^{*} S\right) & \mapsto L^{2}\left(E ;-2 i\left(M-M^{*}\right)\right), \\
G_{*}: L^{2}\left(E ; I-S S^{*}\right) & \mapsto L^{2}\left(E ;-2 i\left(M-M^{*}\right)\right)
\end{aligned}
$$

by the formulae $g \mapsto(M+i I)^{-1} g$ and $g \mapsto\left(M^{*}-i I\right)^{-1} g$ respectively, one arrives at the fact that 
$G F \Phi A_{0}^{(\mathrm{e})} \Phi^{*} F^{*} G^{*}$ and $G_{*} F_{*} \Phi A_{0}^{(\mathrm{e})} \Phi^{*} F_{*}^{*} G_{*}^{*}$ are the operators of multiplication by the independent variable in the space $L^{2}\left(E ;-2 i\left(M-M^{*}\right)\right)$.

Remark 7. The weight $M^{*}-M$ can be assumed to be naturally diagonal in the setting of quantum graphs, see [14] ( $c f$. [18, 19]), including the situation of an infinite number of semi-infinite edges.

The above result only pertains to the absolutely continuous part of the self-adjoint operator $A_{0}$, unlike e.g. the passage to the classical von Neumann direct integral, under which the whole of the self-adjoint operator gets mapped to the multiplication operator in a weighted $L^{2}$-space (see e.g. 6. Chapter 7]). Nevertheless, it proves useful in scattering theory, since it yields an explicit expression

for the scattering matrix $\widehat{\Sigma}$ for the pair $A_{\varkappa}, A_{0}$, which is the image of the scattering operator $\Sigma$ in the spectral representation of the operator $A_{0}$. Namely, we prove the following statement.

Theorem 7.3. The following formula holds:

$$
\widehat{\Sigma}=G F \Sigma(G F)^{*}=(M-\varkappa)^{-1}\left(M^{*}-\varkappa\right)\left(M^{*}\right)^{-1} M,
$$

where the right-hand side represents the operator of multiplication by the corresponding function in the space $L^{2}\left(E ;-2 i\left(M-M^{*}\right)\right)$.

Proof. Using the definition (7.1) of the isometry $F$ along with the relationship (4.19) between $\widetilde{g}$ and $g$ whenever $P_{K}\left(\begin{array}{c}\widetilde{g} \\ g\end{array}\right) \in \Phi \widetilde{N}_{\mathrm{e}}^{\varkappa}$ with $\varkappa=0$, we obtain from (6.9):

$$
F \Sigma F^{*}=\left(I+\chi_{\varkappa}^{-}(S-I)\right)^{-1}\left(I+\chi_{\varkappa}^{+}\left(S^{*}-I\right)\right)\left(I+S^{*}\right)^{-1}(I+S)
$$

where the right-hand side represents the operator of multiplication by the corresponding function.

Furthermore, substituting the expression (2.7) for $S$ in terms of $M$ implies that $F \Sigma F^{*}$ is the operator of multiplication by

$$
(M+i I)(M-\varkappa)^{-1}\left(M^{*}-\varkappa\right)\left(M^{*}\right)^{-1} M(M+i I)
$$

in the space $L^{2}\left(\mathcal{K} ; I-S^{*} S\right)$. Using (7.3), we now obtain the following identity for all $f, g \in$ $L^{2}\left(\mathcal{K} ; I-S^{*} S\right)$ :

$$
\begin{gathered}
\left\langle F \Sigma F^{*} f, g\right\rangle_{L^{2}\left(\mathcal{K} ; I-S^{*} S\right)}=\left\langle\left(I-S^{*} S\right)(M+i I)(M-\varkappa)^{-1}\left(M^{*}-\varkappa\right)\left(M^{*}\right)^{-1} M(M+i I) f, g\right\rangle \\
=\left\langle-2 i\left(M^{*}-i I\right)^{-1}\left(M-M^{*}\right)(M+i I)^{-1}(M+i I)(M-\varkappa)^{-1}\left(M^{*}-\varkappa\right)\left(M^{*}\right)^{-1} M(M+i I) f, g\right\rangle \\
=\left\langle-2 i\left(M-M^{*}\right)(M-\varkappa)^{-1}\left(M^{*}-\varkappa\right)\left(M^{*}\right)^{-1} M(M+i I) f,(M+i I) g\right\rangle,
\end{gathered}
$$

which is equivalent to (7.6), in view of the definition of the operator $G$.

\section{Acknowledgements}

KDC is grateful for the financial support of the Engineering and Physical Sciences Research Council: Grant EP/L018802/2 "Mathematical foundations of metamaterials: homogenisation, dissipation and operator theory". AVK has been partially supported by the RFBR grant 1601-00443-a and the Russian Federation Government megagrant 14.Y26.31.0013. LOS has been partially supported by UNAM-DGAPA-PAPIIT IN105414 and SEP-CONACYT 254062.

We express deep gratitude to Professor Sergey Naboko for his reading the manuscript and making a number of valuable remarks.

We also thank the referee for a number of useful suggestions, which have helped us improve the manuscript. 


\section{Appendix}

\section{Proof of Theorem 4.1,}

We prove Theorem 4.1(i). The proof of Theorem 4.1(ii) is carried out along the same lines.

For any $\left(v_{-}, u, v_{+}\right)$in the space $\mathcal{H}$ given in (4.7), consider the mappings $\mathcal{F}_{ \pm}: \mathcal{H} \rightarrow L^{2}(\mathbb{R}, E)$ introduced in [60, Sec. 2.1] following the corresponding definitions in [48] and given by

$$
\begin{aligned}
& \mathcal{F}_{+}\left(v_{-}, u, v_{+}\right)=-\frac{1}{\sqrt{2 \pi}} \lim _{\epsilon \searrow 0} \alpha \Gamma_{0}\left(A_{\mathrm{i} I}-(\cdot-\mathrm{i} \epsilon) I\right)^{-1} u+S^{*} \hat{v}_{-}+\hat{v}_{+} \\
& \mathcal{F}_{-}\left(v_{-}, u, v_{+}\right)=-\frac{1}{\sqrt{2 \pi}} \lim _{\epsilon \searrow 0} \alpha \Gamma_{0}\left(A_{\mathrm{i} I}^{*}-(\cdot+\mathrm{i} \epsilon) I\right)^{-1} u+\hat{v}_{-}+S \hat{v}_{+},
\end{aligned}
$$

where $\hat{v}_{ \pm}$are the Fourier transforms of $v_{ \pm} \in L^{2}\left(\mathbb{R}_{ \pm}, E\right)$ extended by zero to $L^{2}(\mathbb{R}, E)$. Note that the limits exist almost everywhere due to (4.1).

According to [60, Thm. 2.3], if $\left(\begin{array}{l}\widetilde{g} \\ g\end{array}\right)=\Phi h$, then

$$
\mathcal{F}_{+} h=\widetilde{g}+S^{*} g, \quad \mathcal{F}_{-} h=S \widetilde{g}+g .
$$

Therefore, for proving Theorem 4.1(i), one should establish the validity of the identities:

$$
\mathcal{F}_{ \pm}\left(A_{\varkappa}-z I\right)^{-1} \Phi^{-1}\left(\begin{array}{c}
\widetilde{g} \\
g
\end{array}\right)=\mathcal{F}_{ \pm} \Phi^{-1} P_{K} \frac{1}{\cdot-z}\left(\begin{array}{c}
\widetilde{g} \\
g-\chi_{\varkappa}^{+} \Theta_{\varkappa}^{-1}(z)\left(\widetilde{g}+S^{*} g\right)(z)
\end{array}\right)
$$

for $z \in \mathbb{C}_{-} \cap \rho\left(A_{\varkappa}\right)$. First we compute the left-hand-side of (A.11). It follows from Lemma 3.1(ii), (iii) that, for $z, \lambda \in \mathbb{C}_{-} \cap \rho\left(A_{\varkappa}\right)$ and $h \in \mathcal{H}$,

$$
\begin{aligned}
\alpha \Gamma_{0}\left(A_{\mathrm{i} I}\right. & -z I)^{-1}\left(A_{\varkappa}-\lambda I\right)^{-1} h \\
& =\Theta_{\varkappa}(z) \alpha \Gamma_{0}\left(A_{\varkappa}-z I\right)^{-1}\left(A_{\varkappa}-\lambda I\right)^{-1} h \\
& =\frac{1}{z-\lambda} \Theta_{\varkappa}(z) \alpha \Gamma_{0}\left[\left(A_{\varkappa}-z I\right)^{-1}-\left(A_{\varkappa}-\lambda I\right)^{-1}\right] h \\
& =\frac{1}{z-\lambda}\left[\alpha \Gamma_{0}\left(A_{\mathrm{i} I}-z I\right)^{-1}-\Theta_{\varkappa}(z) \alpha \Gamma_{0}\left(A_{\varkappa}-\lambda I\right)^{-1}\right] h \\
& =\frac{1}{z-\lambda}\left[\alpha \Gamma_{0}\left(A_{\mathrm{i} I}-z I\right)^{-1}-\Theta_{\varkappa}(z) \Theta_{\varkappa}^{-1}(\lambda) \alpha \Gamma_{0}\left(A_{\mathrm{i} I}-\lambda I\right)^{-1}\right] h .
\end{aligned}
$$

Let $z=k-\mathrm{i} \epsilon$ with $k \in \mathbb{R}$, then it follows from the computation above that

$$
\begin{aligned}
& \lim _{\epsilon \searrow 0} \alpha \Gamma_{0}\left(A_{\mathrm{i} I}-(k-\mathrm{i} \epsilon) I\right)^{-1}\left(A_{\varkappa}-\lambda I\right)^{-1} h \\
& =\lim _{\epsilon \searrow 0} \frac{1}{(k-\mathrm{i} \epsilon)-\lambda}\left[\alpha \Gamma_{0}\left(A_{\mathrm{i} I}-(k-\mathrm{i} \epsilon) I\right)^{-1}-\Theta_{\varkappa}(k-\mathrm{i} \epsilon) \Theta_{\varkappa}^{-1}(\lambda) \alpha \Gamma_{0}\left(A_{\mathrm{i} I}-\lambda I\right)^{-1}\right] h .
\end{aligned}
$$

Substituting (A.8) into the last equality, one has

$$
\mathcal{F}_{+}\left(A_{\varkappa}-\lambda I\right)^{-1} h=\frac{1}{\cdot-\lambda}\left[\mathcal{F}_{+} h-\Theta_{\varkappa}(\cdot) \Theta_{\varkappa}^{-1}(\lambda) \mathcal{F}_{+} h(\lambda)\right] .
$$

Hence, in view of (A.10), one concludes

$$
\mathcal{F}_{+}\left(A_{\varkappa}-\lambda I\right)^{-1} \Phi^{-1}\left(\begin{array}{l}
\widetilde{g} \\
g
\end{array}\right)=\frac{1}{\cdot-\lambda}\left[\widetilde{g}+S^{*} g-\Theta_{\varkappa}(\cdot) \Theta_{\varkappa}^{-1}(\lambda)\left(\widetilde{g}+S^{*} g\right)(\lambda)\right] .
$$

On the basis of Lemma 3.1(iii), (iv) and reasoning in the same fashion as was done to obtain 
A.12, one verifies

$$
\mathcal{F}_{-}\left(A_{\varkappa}-\lambda I\right)^{-1} \Phi^{-1}\left(\begin{array}{l}
\widetilde{g} \\
g
\end{array}\right)=\frac{1}{\cdot-\lambda}\left[S \widetilde{g}+g-\widehat{\Theta}_{\varkappa}(\cdot) \Theta_{\varkappa}^{-1}(\lambda)\left(\widetilde{g}+S^{*} g\right)(\lambda)\right] .
$$

Let us focus on the right hand side of (A.11). Note that

$$
\begin{aligned}
& P_{K} \frac{1}{\cdot-z}\left(\begin{array}{c}
\widetilde{g} \\
g-\chi_{\varkappa}^{+} \Theta_{\varkappa}^{-1}(z)\left(\widetilde{g}+S^{*} g\right)(z)
\end{array}\right) \\
& =\left(\begin{array}{c}
\frac{\widetilde{g}}{--z}-P_{+} \frac{1}{\cdot-z}\left[\widetilde{g}+S^{*} g-S^{*} \chi_{\varkappa}^{+} \Theta_{\varkappa}^{-1}(z)\left(\widetilde{g}+S^{*} g\right)(z)\right] \\
\frac{1}{\cdot-z}\left(g-\chi_{\varkappa}^{+} \Theta_{\varkappa}^{-1}(z)\left(\widetilde{g}+S^{*} g\right)(z)\right)-P_{-} \frac{1}{--z}\left[S \widetilde{g}+g-\chi_{\varkappa}^{+} \Theta_{\varkappa}^{-1}(z)\left(\widetilde{g}+S^{*} g\right)(z)\right]
\end{array}\right) \\
& =\frac{1}{\cdot-z}\left(\begin{array}{c}
\widetilde{g}-\left(\widetilde{g}+S^{*} g\right)(z)+S^{*}(\bar{z}) \chi_{\varkappa}^{+} \Theta_{\varkappa}^{-1}(z)\left(\widetilde{g}+S^{*} g\right)(z) \\
g-\chi_{\varkappa}^{+} \Theta_{\varkappa}^{-1}(z)\left(\widetilde{g}+S^{*} g\right)(z)
\end{array}\right)
\end{aligned}
$$

where (4.6) is used in the first equality and in the second the fact that if $f$ is a function in $H_{-}^{2}$, then, for any $z \in \mathbb{C}_{-}$,

$$
P_{+}\left(\frac{f}{\cdot-z}\right)=P_{+}\left(\frac{f+f(z)-f(z)}{\cdot-z}\right)=P_{+}\left(\frac{f(z)}{\cdot-z}\right)=\frac{f(z)}{\cdot-z} .
$$

Now, apply $\mathcal{F}_{+} \Phi^{-1}$ to (A.14) taking into account (A.10):

$$
\begin{aligned}
& \mathcal{F}_{+} \Phi^{-1} \frac{1}{\cdot-z}\left(\begin{array}{c}
\widetilde{g}-\left(\widetilde{g}+S^{*} g\right)(z)+S^{*}(\bar{z}) \chi_{\varkappa}^{+} \Theta_{\varkappa}^{-1}(z)\left(\widetilde{g}+S^{*} g\right)(z) \\
g-\chi_{\varkappa}^{+} \Theta_{\varkappa}^{-1}(z)\left(\widetilde{g}+S^{*} g\right)(z)
\end{array}\right) \\
& =\frac{1}{\cdot-z}\left[\widetilde{g}+S^{*} g-\left(\widetilde{g}+S^{*} g\right)(z)+\left(S^{*}(\bar{z})-S^{*}\right) \chi_{\varkappa}^{+} \Theta_{\varkappa}^{-1}(z)\left(\widetilde{g}+S^{*} g\right)(z)\right] \\
& =\frac{1}{\cdot-z}\left[\widetilde{g}+S^{*} g-\left(\Theta_{\varkappa}(z)-\left(S^{*}(\bar{z})-S^{*}\right) \chi_{\varkappa}^{+}\right) \Theta_{\varkappa}^{-1}(z)\left(\widetilde{g}+S^{*} g\right)(z)\right] \\
& =\frac{1}{\cdot-z}\left[\widetilde{g}+S^{*} g-\Theta(\cdot) \Theta_{\varkappa}^{-1}(z)\left(\widetilde{g}+S^{*} g\right)(z)\right] .
\end{aligned}
$$

By combining the last equality with A.12), we have established the first identity in (A.11).

Now, if one applies $\mathcal{F}_{-} \Phi^{-1}$ to (A.14), then, in view of (A.10), one has

$$
\begin{aligned}
& \mathcal{F}_{-} \Phi^{-1} \frac{1}{\cdot-z}\left(\begin{array}{c}
\widetilde{g}-\left(\widetilde{g}+S^{*} g\right)(z)+S^{*}(\bar{z}) \chi_{\varkappa}^{+} \Theta_{\varkappa}^{-1}(z)\left(\widetilde{g}+S^{*} g\right)(z) \\
g-\chi_{\varkappa}^{+} \Theta_{\varkappa}^{-1}(z)\left(\widetilde{g}+S^{*} g\right)(z)
\end{array}\right) \\
& =\frac{1}{\cdot-z}\left[S \widetilde{g}+g-S\left(\widetilde{g}+S^{*} g\right)(z)-\left(I-S S^{*}(\bar{z})\right) \chi_{\varkappa}^{+} \Theta_{\varkappa}^{-1}(z)\left(\widetilde{g}+S^{*} g\right)(z)\right] \\
& =\frac{1}{\cdot-z}\left[S \widetilde{g}+g-\left(S \Theta_{\varkappa}(z)+\chi_{\varkappa}^{+}-S S^{*}(\bar{z}) \chi_{\varkappa}^{+}\right) \Theta_{\varkappa}^{-1}(z)\left(\widetilde{g}+S^{*} g\right)(z)\right] \\
& =\frac{1}{\cdot-z}\left[S \widetilde{g}+g-\left(S \chi_{\varkappa}^{-}+\chi_{\varkappa}^{-}\right) \Theta_{\varkappa}^{-1}(z)\left(\widetilde{g}+S^{*} g\right)(z)\right] \\
& =\frac{1}{\cdot-z}\left[S \widetilde{g}+g-\widehat{\Theta}_{\varkappa}(\cdot) \Theta_{\varkappa}^{-1}(z)\left(\widetilde{g}+S^{*} g\right)(z)\right]
\end{aligned}
$$

Thus, after comparing this last equality with (A.13), we arrive at the second identity in (A.11).

\section{Proof of Theorem 4.2 ,}


Let us first show that the following inclusion holds

$$
\mathfrak{N}_{ \pm}^{\varkappa} \subset\left\{\left(\begin{array}{c}
\widetilde{g} \\
g
\end{array}\right) \in \mathfrak{H}: \Phi\left(A_{\varkappa}-z I\right)^{-1} \Phi^{*} P_{K}\left(\begin{array}{l}
\widetilde{g} \\
g
\end{array}\right)=P_{K} \frac{1}{\cdot-z}\left(\begin{array}{l}
\widetilde{g} \\
g
\end{array}\right) \text { for all } z \in \mathbb{C}_{ \pm}\right\}
$$

Consider $z \in \mathbb{C}_{-} \cap \rho\left(A_{\varkappa}\right)$. By (4.6) and Theorem 4.1 one has

$$
\begin{aligned}
& \Phi\left(A_{\varkappa}-z I\right)^{-1} \Phi^{-1} P_{K}\left(\begin{array}{c}
\widetilde{g} \\
g
\end{array}\right)=\Phi\left(A_{\varkappa}-z I\right)^{-1} \Phi^{-1}\left(\begin{array}{c}
\widetilde{g}-P_{+}\left(\widetilde{g}+S^{*} g\right) \\
g-P_{-}(S \widetilde{g}+g)
\end{array}\right) \\
& =P_{K} \frac{1}{\cdot-z}\left(\begin{array}{c}
\widetilde{g}-P_{+}\left(\widetilde{g}+S^{*} g\right) \\
g-P_{-}(S \widetilde{g}+g)-\chi_{\varkappa}^{+} \Theta_{\varkappa}^{-1}(z)\left[\widetilde{g}-P_{+}\left(\widetilde{g}+S^{*} g\right)+S^{*}\left(g-P_{-}(S \widetilde{g}+g)\right)\right](z)
\end{array}\right)
\end{aligned}
$$

where

$$
\left[\widetilde{g}-P_{+}\left(\widetilde{g}+S^{*} g\right)+S^{*}\left(g-P_{-}(S \widetilde{g}+g)\right)\right](z)
$$

is to be understood as the analytic continuation into the lower half-plane of the function

$$
\widetilde{g}-P_{+}\left(\widetilde{g}+S^{*} g\right)+S^{*}\left(g-P_{-}(S \widetilde{g}+g)\right)=P_{-}\left(\widetilde{g}+S^{*} g\right)-S^{*} P_{-}(S \widetilde{g}+g),
$$

which is clearly an element of $H_{-}^{2}(E)$. Thus,

$$
\Phi\left(A_{\varkappa}-z I\right)^{-1} \Phi^{-1} P_{K}\left(\begin{array}{l}
\widetilde{g} \\
g
\end{array}\right)=P_{K} \frac{1}{\cdot-z}\left(\begin{array}{c}
\widetilde{g}-P_{+}\left(\widetilde{g}+S^{*} g\right) \\
g-P_{-}(S \widetilde{g}+g)-\gamma(z)
\end{array}\right)
$$

where

$$
\gamma(z):=\chi_{\varkappa}^{+} \Theta_{\varkappa}^{-1}(z)\left(P_{-}\left(\widetilde{g}+S^{*} g\right)(z)-S^{*} P_{-}(S \widetilde{g}+g)(z)\right) .
$$

The following lemma is needed to simplify the form of $\gamma(z)$.

Lemma A.1. For all $\left(\begin{array}{c}\widetilde{g} \\ g\end{array}\right) \in \mathfrak{H}$ the following identity holds:

$$
\gamma(z)=-P_{-}(S \widetilde{g}+g)(z) \quad \forall z \in \mathbb{C}_{-} .
$$

Proof.

$$
\begin{gathered}
\chi_{\varkappa}^{+} \Theta_{\varkappa}^{-1}(z)\left(P_{-}\left(\widetilde{g}+S^{*} g\right)(z)-S^{*}(\bar{z}) P_{-}(S \widetilde{g}+g)(z)\right) \\
=\chi_{\varkappa}^{+}\left(I+i \alpha\left(B_{\varkappa}-M(z)\right)^{-1} \alpha \chi_{\varkappa}^{+}\right)\left(P_{-}\left(\widetilde{g}+S^{*} g\right)(z)-S^{*}(\bar{z}) P_{-}(S \widetilde{g}+g)(z)\right) \\
=\left(I+i \chi_{\varkappa}^{+} \alpha\left(B_{\varkappa}-M(z)\right)^{-1} \alpha\right) \chi_{\varkappa}^{+}\left(P_{-}\left(\widetilde{g}+S^{*} g\right)(z)-S^{*}(\bar{z}) P_{-}(S \widetilde{g}+g)(z)\right) \\
=\left(I+\chi_{\varkappa}^{+}\left(S^{*}(\bar{z})-I\right)\right)^{-1}\left(\chi_{\varkappa}^{+} P_{-}\left(\widetilde{g}+S^{*} g\right)(z)-\chi_{\varkappa}^{+} S^{*}(\bar{z}) P_{-}(S \widetilde{g}+g)(z)\right) \\
=\left(I+\chi_{\varkappa}^{+}\left(S^{*}(\bar{z})-I\right)\right)^{-1}\left(-\chi_{\varkappa}^{-} P_{-}(S \widetilde{g}+g)(z)-\chi_{\varkappa}^{+} S^{*}(\bar{z}) P_{-}(S \widetilde{g}+g)(z)\right) \\
=\left(I+\chi_{\varkappa}^{+}\left(S^{*}(\bar{z})-I\right)\right)^{-1}\left(-\chi_{\varkappa}^{-}-\chi_{\varkappa}^{+} S^{*}(\bar{z})\right) P_{-}(S \widetilde{g}+g)(z)=-P_{-}(S \widetilde{g}+g)(z),
\end{gathered}
$$

where we use the fact that

$$
I+i \chi_{\varkappa}^{+} \alpha\left(B_{\varkappa}-M(z)\right)^{-1} \alpha=\left(I+\chi_{\varkappa}^{+}\left(S^{*}(\bar{z})-I\right)\right)^{-1},
$$

proved in a similar way to (3.9). 
Therefore, for $\left(\begin{array}{l}\widetilde{g} \\ g\end{array}\right) \in \mathfrak{N}_{-}^{\varkappa}$ the expression (A.17) can be re-written as

$$
\begin{aligned}
\Phi\left(A_{\varkappa}-z I\right)^{-1} \Phi^{-1} P_{K}\left(\begin{array}{c}
\widetilde{g} \\
g
\end{array}\right) & =P_{K} \frac{1}{\cdot-z}\left(\begin{array}{c}
\widetilde{g}-P_{+}\left(\widetilde{g}+S^{*} g\right) \\
g-P_{-}(S \widetilde{g}+g)+P_{-}(S \widetilde{g}+g)(z)
\end{array}\right) \\
& =P_{K} \frac{1}{\cdot-z}\left[\left(\begin{array}{c}
\widetilde{g} \\
g
\end{array}\right)-\left(\begin{array}{c}
P_{+}\left(\widetilde{g}+S^{*} g\right) \\
P_{-}(S \widetilde{g}+g)-P_{-}(S \widetilde{g}+g)(z)
\end{array}\right)\right]
\end{aligned}
$$

One completes the proof by observing that

$$
\frac{P_{+}\left(\widetilde{g}+S^{*} g\right)}{\cdot-z} \in H_{+}^{2}(E), \quad \frac{P_{-}(S \widetilde{g}+g)-P_{-}(S \widetilde{g}+g)(z)}{\cdot-z} \in H_{-}^{2}(E) .
$$

We have thus shown that

$$
\mathfrak{N}_{-}^{\varkappa} \subset\left\{\left(\begin{array}{l}
\widetilde{g} \\
g
\end{array}\right) \in \mathfrak{H}: \Phi\left(A_{\varkappa}-z I\right)^{-1} \Phi^{*} P_{K}\left(\begin{array}{l}
\widetilde{g} \\
g
\end{array}\right)=P_{K} \frac{1}{\cdot-z}\left(\begin{array}{l}
\widetilde{g} \\
g
\end{array}\right) \text { for all } z \in \mathbb{C}_{-}\right\} .
$$

The inclusion

$$
\mathfrak{N}_{+}^{\varkappa} \subset\left\{\left(\begin{array}{l}
\widetilde{g} \\
g
\end{array}\right) \in \mathfrak{H}: \Phi\left(A_{\varkappa}-z I\right)^{-1} \Phi^{*} P_{K}\left(\begin{array}{l}
\widetilde{g} \\
g
\end{array}\right)=P_{K} \frac{1}{\cdot-z}\left(\begin{array}{l}
\widetilde{g} \\
g
\end{array}\right) \text { for all } z \in \mathbb{C}_{+}\right\}
$$

is proved analogously.

To prove the converse inclusion, i.e.

$$
\left\{\left(\begin{array}{l}
\widetilde{g} \\
g
\end{array}\right) \in \mathfrak{H}: \Phi\left(A_{\varkappa}-z I\right)^{-1} \Phi^{*} P_{K}\left(\begin{array}{l}
\widetilde{g} \\
g
\end{array}\right)=P_{K} \frac{1}{\cdot-z}\left(\begin{array}{l}
\widetilde{g} \\
g
\end{array}\right) \text { for all } z \in \mathbb{C}_{ \pm}\right\} \subset \mathfrak{N}_{ \pm}^{\varkappa}
$$

one again follows the arguments of [48, Thm. 4]. According to (A.17), for all $z \in \mathbb{C}_{-} \cap \rho\left(A_{\varkappa}\right)$, one has

$$
\Phi\left(A_{\varkappa}-z I\right)^{-1} \Phi^{-1} P_{K}\left(\begin{array}{c}
\widetilde{g} \\
g
\end{array}\right)=P_{K} \frac{1}{\cdot-z}\left(\begin{array}{c}
\widetilde{g}-P_{+}\left(\widetilde{g}+S^{*} g\right) \\
g-P_{-}(S \widetilde{g}+g)-\gamma(z)
\end{array}\right),
$$

where $\gamma(z)$ is defined in (A.18). Denoting $\widehat{\gamma}:=\gamma+P_{-}(S \widetilde{g}+g)$, it follows from (4.6) that

$$
\begin{aligned}
\Phi\left(A_{\varkappa}-z I\right)^{-1} \Phi^{-1} P_{K}\left(\begin{array}{c}
\widetilde{g} \\
g
\end{array}\right)-P_{K} \frac{1}{\cdot-z}\left(\begin{array}{l}
\widetilde{g} \\
g
\end{array}\right) & =P_{K}\left(\begin{array}{c}
0 \\
-\widehat{\gamma}(z)(\cdot-z)^{-1}
\end{array}\right) \\
& =\left(\begin{array}{c}
P_{+}\left(S^{*} \widehat{\gamma}(z)(\cdot-z)^{-1}\right) \\
-\widehat{\gamma}(z)(\cdot-z)^{-1}+P_{-}\left(\widehat{\gamma}(z)(\cdot-z)^{-1}\right)
\end{array}\right)
\end{aligned}
$$

Furthermore, in view of A.15), one has

$$
P_{+}\left[\frac{S^{*} \widehat{\gamma}(z)}{\cdot-z}\right]=\frac{S^{*}(\bar{z}) \widehat{\gamma}(z)}{\cdot-z}
$$

and, clearly,

$$
P_{-}\left[\frac{\widehat{\gamma}(z)}{\cdot-z}\right]=0
$$

Therefore

$$
\Phi\left(A_{\varkappa}-z I\right)^{-1} \Phi^{-1} P_{K}\left(\begin{array}{l}
\widetilde{g} \\
g
\end{array}\right)-P_{K} \frac{1}{\cdot-z}\left(\begin{array}{l}
\widetilde{g} \\
g
\end{array}\right)=\left(\begin{array}{c}
S^{*}(\bar{z}) \widehat{\gamma}(z)(\cdot-z)^{-1} \\
-\widehat{\gamma}(z)(\cdot-z)^{-1}
\end{array}\right) .
$$

Since

$$
\Phi\left(A_{\varkappa}-z I\right)^{-1} \Phi^{*} P_{K}\left(\begin{array}{c}
\widetilde{g} \\
g
\end{array}\right)=P_{K} \frac{1}{\cdot-z}\left(\begin{array}{c}
\widetilde{g} \\
g
\end{array}\right) \text { for all } z \in \mathbb{C}_{-},
$$


one has

$$
\left(\begin{array}{c}
S^{*}(\bar{z}) \widehat{\gamma}(z)(\cdot-z)^{-1} \\
-\widehat{\gamma}(z)(\cdot-z)^{-1}
\end{array}\right)=0
$$

which in its turn implies

$$
\left(S^{*}-S^{*}(\bar{z})\right) \widehat{\gamma}(z)(\cdot-z)^{-1}=0 .
$$

From this equality, by virtue of the assumption that $\operatorname{ker}(\alpha)=\{0\}$, one obtains that $\gamma(z)=0$ for all $z \in \mathbb{C}_{-} \cap \rho\left(A_{\varkappa}\right)$ (see details in the proof of [47, Lem.4]). Taking into account the definition of $\widehat{\gamma}$, one arrives at

$$
\chi_{\varkappa}^{-} P_{ \pm}(S \widetilde{g}+g)+\chi_{\varkappa}^{+} P_{ \pm}\left(\widetilde{g}+S^{*} g\right)=0
$$

The inclusion

$$
\left\{\left(\begin{array}{l}
\widetilde{g} \\
g
\end{array}\right) \in \mathfrak{H}: \Phi\left(A_{\varkappa}-z I\right)^{-1} \Phi^{*} P_{K}\left(\begin{array}{l}
\widetilde{g} \\
g
\end{array}\right)=P_{K} \frac{1}{\cdot-z}\left(\begin{array}{l}
\widetilde{g} \\
g
\end{array}\right) \text { for all } z \in \mathbb{C}_{+}\right\} \subset \mathfrak{N}_{+}^{\varkappa}
$$

is proved in a similar way.

\section{Proof of Theorem 4.3 ,}

To prove the inclusion

$$
\widetilde{N}_{-}^{\varkappa} \subset\left\{u \in \mathcal{H}: \chi_{\varkappa}^{+} \alpha \Gamma_{0}\left(A_{\varkappa}-z I\right)^{-1} u \in H_{-}^{2}(E)\right\},
$$

one has to show that $u \in \Phi^{*} P_{K} \mathfrak{N}_{-}^{\varkappa}$ implies $\chi_{\varkappa}^{+} \alpha \Gamma_{0}\left(A_{\varkappa}-z I\right)^{-1} u \in H_{-}^{2}(E)$. By (4.6), if $u=$ $\Phi^{*} P_{K}\left(\begin{array}{l}\widetilde{g} \\ g\end{array}\right)$, then

$$
\Phi u=\left(\begin{array}{c}
\widetilde{g}-P_{+}\left(\widetilde{g}+S^{*} g\right) \\
g-P_{-}(S \widetilde{g}+g)
\end{array}\right) .
$$

Thus, in view of the inclusion $\left(\begin{array}{l}\widetilde{g} \\ g\end{array}\right) \in K$, it follows from (A.10) that

$$
\begin{aligned}
\mathcal{F}_{+} u & =\widetilde{g}-P_{+}\left(\widetilde{g}+S^{*} g\right)+S^{*} g-S^{*} P_{-}(S \widetilde{g}+g) \\
& =\left(I-P_{+}\right)\left(\widetilde{g}+S^{*} g\right)-S^{*} P_{-}(S \widetilde{g}+g) \\
& =P_{-}\left(\widetilde{g}+S^{*} g\right)-S^{*} P_{-}(S \widetilde{g}+g) .
\end{aligned}
$$

By analytic continuation of $\mathcal{F}_{+} u$ into the lower half-plane, taking into account (A.8), one arrives at

$$
\alpha \Gamma_{0}\left(A_{\mathrm{i} I}-z I\right)^{-1} u=-\sqrt{2 \pi}\left(P_{-}\left(\widetilde{g}+S^{*} g\right)(z)-S^{*}(\bar{z}) P_{-}(S \widetilde{g}+g)(z)\right) \quad \forall z \in \mathbb{C}_{-} .
$$

Combining this with Lemma 3.1(iii), we write

$$
\alpha \Gamma_{0}\left(A_{\varkappa}-z I\right)^{-1} u=-\sqrt{2 \pi} \Theta_{\varkappa}^{-1}(z)\left(P_{-}\left(\widetilde{g}+S^{*} g\right)(z)-S^{*}(\bar{z}) P_{-}(S \widetilde{g}+g)(z)\right) .
$$

Finally, using Lemma A.1 from the proof of Theorem 4.2 above, we obtain

$$
\chi_{\varkappa}^{+} \alpha \Gamma_{0}\left(A_{\varkappa}-z I\right)^{-1} u=\sqrt{2 \pi} P_{-}(S \widetilde{g}+g)(z),
$$

To demonstrate the converse inclusion

$$
\left\{u \in \mathcal{H}: \chi_{\varkappa}^{+} \alpha \Gamma_{0}\left(A_{\varkappa}-z I\right)^{-1} u \in H_{-}^{2}(E)\right\} \subset \widetilde{N}_{-}^{\varkappa},
$$

we show that, whenever $\chi_{\varkappa}^{+} \alpha \Gamma_{0}\left(A_{\varkappa}-z I\right)^{-1} u \in H_{-}^{2}(E)$, the vector

$$
\left(\begin{array}{l}
\widetilde{g} \\
g
\end{array}\right)=\Phi u-\frac{1}{2 \pi}\left(\begin{array}{c}
0 \\
\alpha \Gamma_{0}\left(A_{\varkappa}-z I\right)^{-1} u
\end{array}\right)
$$


satisfies

$$
P_{-}\left(\chi_{\varkappa}^{+}\left(\widetilde{g}+S^{*} g\right)+\chi_{\varkappa}^{-}(S \widetilde{g}+g)\right)=0,
$$

and hence $u=\Phi^{*} P_{K}\left(\begin{array}{c}\widetilde{g} \\ g\end{array}\right) \in \Phi^{*} P_{K} \mathfrak{N}_{-}^{\varkappa}=\widetilde{N}_{\mathrm{e}}^{\varkappa}$. Indeed, introducing the notation

$$
\Phi u=:\left(\begin{array}{c}
\widetilde{g_{0}} \\
g_{0}
\end{array}\right), \quad h^{-}:=\frac{1}{2 \pi} \alpha \Gamma_{0}\left(A_{i I}-z I\right)^{-1} u,
$$

we have

$$
\begin{gathered}
P_{-}\left(\chi_{\varkappa}^{+}\left(\widetilde{g_{0}}+S^{*}\left(g_{0}+h^{-}\right)\right)+\chi_{\varkappa}^{-}\left(S \widetilde{g_{0}}+g_{0}+h^{-}\right)\right) \\
=\chi_{\varkappa}^{+}\left(\widetilde{g_{0}}+S^{*} g_{0}\right)-P_{+} \chi_{\varkappa}^{+}\left(\widetilde{g_{0}}+S^{*} g_{0}\right)+P_{-} \chi_{\varkappa}^{-}\left(S \widetilde{g_{0}}+g_{0}\right)+\left(I+\chi_{\varkappa}^{+}\left(S^{*}-I\right)\right) h^{-} \\
=\chi_{\varkappa}^{+} \mathcal{F}_{+} u+\left(I+\chi_{\varkappa}^{+}\left(S^{*}-I\right)\right) h^{-},
\end{gathered}
$$

By the analytic continuation into the lower half-plane and using Lemma 3.1(i), it follows that (A.19) represents the boundary value on the real line of the function

$$
\begin{gathered}
-\frac{1}{2 \pi} \chi_{\varkappa}^{+} \alpha \Gamma_{0}\left(A_{i I}-z I\right)^{-1} u+\left(I+\chi_{\varkappa}^{+}\left(S^{*}(\bar{z})-I\right)\right) h^{-}(z) \\
=-\frac{1}{2 \pi} \chi_{\varkappa}^{+} \Theta_{\varkappa}(z) \alpha \Gamma_{0}\left(A_{\varkappa}-z I\right)^{-1} u+\left(I+\chi_{\varkappa}^{+}\left(S^{*}(\bar{z})-I\right)\right) h^{-}(z) \\
=\left(I+\chi_{\varkappa}^{+}\left(S^{*}(\bar{z})-I\right)\right)\left(h^{-}(z)-\frac{1}{2 \pi} \chi_{\varkappa}^{+} \alpha \Gamma_{0}\left(A_{\varkappa}-z I\right)^{-1} u\right)=0,
\end{gathered}
$$

where in order to pass from (A.20) to (A.21) we have used the fact that (see (3.1))

$$
\chi_{\varkappa}^{+} \Theta_{\varkappa}(z)=\left(I-\mathrm{i} \chi_{\varkappa}^{+} \alpha\left(B_{\mathrm{i} I}-M(z)\right)^{-1} \alpha\right) \chi_{\varkappa}^{+}=\left(I+\chi_{\varkappa}^{+}\left(S^{*}(\bar{z})-I\right)\right) \chi_{\varkappa}^{+}, \quad z \in \mathbb{C}_{-} .
$$

Hence, the expression (A.19) vanishes, which concludes the proof.

The property

$$
\tilde{N}_{+}^{\varkappa}=\left\{u \in \mathcal{H}: \chi_{\varkappa}^{-} \alpha \Gamma_{0}\left(A_{\varkappa}-z I\right)^{-1} u \in H_{+}^{2}(E)\right\}
$$

is proved in a similar way.

\section{Proof of Proposition 5.1 .}

Suppose that $z \in \mathbb{C}_{+}$. If

$$
\int_{\mathbb{R}} \frac{d \mu(s)}{s-z} \in H_{+}^{2},
$$

then, by [58, Thm. 5.19], there exists a function $f \in L^{2}(\mathbb{R})$ such that

$$
\int_{\mathbb{R}} \frac{f(s) d s-d \mu(s)}{s-z}=0 .
$$

Fix a $z_{0} \in \mathbb{C}_{+}$, then

$$
\begin{aligned}
0 & =\int_{\mathbb{R}} \frac{f(s) d s-d \mu(s)}{s-z}-\int_{\mathbb{R}} \frac{f(s) d s-d \mu(s)}{s-z_{0}} \\
& =\left(z-z_{0}\right) \int_{\mathbb{R}} \frac{f(s) d s-d \mu(s)}{(s-z)\left(s-z_{0}\right)} .
\end{aligned}
$$

Thus, one has

$$
\int_{\mathbb{R}} \frac{1}{s-z} \frac{f(s) d s-d \mu(s)}{s-z_{0}}=0, \quad \text { for all } z \in \mathbb{C}_{+} \backslash\left\{z_{0}\right\}
$$


where now $\left(s-z_{0}\right)^{-1}(f(s) d s-d \mu(s))$ is a complex measure on $\mathbb{R}$. Further, we invoke to the upper half-plane counterpart of the theorem by F. and M. Riesz obtained by applying the conformal mapping from the unit circle onto the upper half plane [34, Chap. 2, Sec. A]. This theorem implies that $\left(s-z_{0}\right)^{-1}(f(s) d t-d \mu(s))$ is absolutely continuous with respect to the Lebesgue measure and, therefore, the same applies to $d \mu(s)$.

The case of $H_{-}^{2}$ is treated likewise.

\section{References}

[1] V. M. Adamjan, D. Z. Arov. Unitary couplings of semi-unitary operators. (Russian) Mat. Issled., 1(2):3-64, 1966; English translation in Amer. Math Soc. Transl. Ser. 2, 95, 1970

[2] Adamyan, V. M.; Pavlov, B. S. Zero-radius potentials and M. G. Kreŭn's formula for generalized resolvents. (Russian); translated from Zap. Nauchn. Sem. Leningrad. Otdel. Mat. Inst. Steklov. (LOMI) 149 (1986), Issled. Lineऍ̆n. Teor. Funktsiǔ. XV, 7-23, 186 J. Soviet Math. 42 (1986), no. 2, 1537-1550

[3] Amrein, W. O.; Pearson, D. B. $M$ operators: a generalisation of Weyl-Titchmarsh theory. J. Comput. Appl. Math. 171, no. 1-2: 1-26, 2004.

[4] M. Š. Birman Existence conditions for wave operators. (Russian) Izv. Akad. Nauk SSSR Ser. Mat., 27: 883-906, 1963.

[5] M. Š. Birman, M. G. Kreı̆n. On the theory of wave operators and scattering operators. (Russian) Dokl. Akad. Nauk SSSR 144:475-478, 1962.

[6] M. Š. Birman and M. Z. Solomjak. Spectral theory of selfadjoint operators in Hilbert space. Mathematics and its Applications (Soviet Series). D. Reidel Publishing Co., Dordrecht, 1987. Translated from the 1980 Russian original by S. Khrushchëv and V. Peller.

[7] G. Borg. Eine Umkehrung der Sturm-Liouvilleschen Eigenwertaufgabe. Bestimmung der Differentialgleichung durch die Eigenwerte. (German) Acta Math. 78:1-96, 1946.

[8] G. Borg. Uniqueness theorems in the spectral theory of $y^{\prime \prime}+(\lambda-q(x)) y=0$. In Den 11te Skandinaviske Matematikerkongress, Trondheim, 1949, pages 276-287. Johan Grundt Tanums Forlag, Oslo, 1952.

[9] M. S. Brodskij. Triangular and Jordan representations of linear operators, Translations of Mathematical Monographs. Vol. 32. Providence, R.I.: American Mathematical Society, 1971.

[10] M. Brown, M. Marletta, S. Naboko, and I. Wood. Boundary triples and $M$-functions for non-selfadjoint operators, with applications to elliptic PDEs and block operator matrices. $J$. Lond. Math. Soc. (2), 77(3):700-718, 2008.

[11] M. Brown, M. Marletta, S. Naboko, and I. Wood. The functional model for maximal dissipative operators: An approach in the spirit of operator knots, 29 pp., arXiv:1804.08963.

[12] K. D. Cherednichenko, Yu. Yu. Ershova, and A. V. Kiselev, 2019. Time-dispersive behaviour as a feature of critical contrast media, SIAM J. Appl. Math. 79(2), 690-715.

[13] K. Cherednichenko, Y. Ershova, A. Kiselev, and S. Naboko, 2019. Unified approach to criticalcontrast homogenisation with explicit links to time-dispersive media. Trans. Moscow Math. Soc. 80(2), 295-342.

[14] K. D. Cherednichenko, A. V. Kiselev, L. O. Silva. Functional model for extensions of symmetric operators and applications to scattering theory. Netw. and Heterog. Media, 13(2): 191-215, 2018. 
[15] P. Deift, E. Trubowitz. Inverse scattering on the line. Comm. Pure Appl. Math. 32(2):121-251, 1979.

[16] V. Derkach. Boundary triples, Weyl functions, and the Kreŭn formula. Operator Theory: Living Reference Work, DOI 10.1007/978-3-0348-0692-3_32-1 Springer Basel, 2015

[17] V. A. Derkach and M. M. Malamud. Generalized resolvents and the boundary value problems for Hermitian operators with gaps. J. Funct. Anal., 95(1):1-95, 1991.

[18] Y. Ershova, I. I. Karpenko, and A. V. Kiselev. Isospectrality for graph Laplacians under the change of coupling at graph vertices. J. Spectr. Theory, 6(1):43-66, 2016.

[19] Y. Ershova, I. I. Karpenko, and A. V. Kiselev. Isospectrality for graph Laplacians under the change of coupling at graph vertices: necessary and sufficient conditions. Mathematika, 62(1):210-242, 2016.

[20] L. D. Faddeyev. The inverse problem in the quantum theory of scattering. J. Mathematical Phys., 4:72-104, 1963.

[21] L. D. Faddeev The inverse problem in the quantum theory of scattering. II. (Russian) Current problems in mathematics, Vol. 3 (Russian), Akad. Nauk SSSR Vsesojuz. Inst. Naučn. i Tehn. Informacii, Moscow, 93-180, 1974. English translation in:J. Sov. Math., 5: 334-396, 1976.

[22] K. O. Friedrichs. On the perturbation of continuous spectra. Communications on Appl. Math. 1: 361-406, 1948.

[23] I. M. Gel'fand, B. M. Levitan. On the determination of a differential equation from its spectral function. (Russian) Izvestiya Akad. Nauk SSSR. Ser. Mat. 15:309-360, 1951.

[24] Gohberg, I. C., Krein, M. G., Introduction to the theory of linear nonself-adjoint operators, Translations of Mathematical Monographs, Vol. 18. AMS, Providence, R.I., 1969.

[25] M. L. Gorbachuk and V. I. Gorbachuk. The theory of selfadjoint extensions of symmetric operators; entire operators and boundary value problems. Ukraïn. Mat. Zh., 46(1-2):55-62, 1994.

[26] M. L. Gorbachuk and V. I. Gorbachuk. M. G. Krein's lectures on entire operators, volume 97 of Operator Theory: Advances and Applications. Birkhäuser Verlag, Basel, 1997.

[27] V. I. Gorbachuk and M. L. Gorbachuk. Boundary value problems for operator differential equations, volume 48 of Mathematics and its Applications (Soviet Series). Kluwer Academic Publishers Group, Dordrecht, 1991. Translated and revised from the 1984 Russian original.

[28] I. Kac and M. G. Krĕn. $R$-functions-analytic functions mapping upper half-plane into itself. Amer. Math. Soc. Transl. Series 2, 103:1-18, 1974.

[29] T. Kato. Perturbation theory for linear operators. Springer-Verlag, Berlin, Second edition, 1976. Grundlehren der Mathematischen Wissenschaften, Band 132.

[30] T. Kato and S. T. Kuroda. The abstract theory of scattering. Rocky Mountain J. Math., 1(1):127-171, 1971.

[31] A. V. Kiselev. Similarity problem for non-self-adjoint extensions of symmetric operators. In Methods of spectral analysis in mathematical physics, volume 186 of Oper. Theory Adv. Appl., pages 267-283. Birkhäuser Verlag, Basel, 2009.

[32] A. N. Kočuber. Extensions of symmetric operators and of symmetric binary relations. Mat. Zametki, 17:41-48, 1975. 
[33] A. N. Kočuber. Characteristic functions of symmetric operators and their extensions (in Russian). Izv. Akad. Nauk Arm. SSR Ser. Mat., 15(3):219-232, 1980

[34] P. Koosis. Introduction to $H_{p}$ spaces, volume 115 of Cambridge Tracts in Mathematics. Cambridge University Press, Cambridge, second edition, 1998. With two appendices by V. P. Havin [Viktor Petrovich Khavin].

[35] V. Kostrykin and R. Schrader. The inverse scattering problem for metric graphs and the traveling salesman problem. Preprint arXiv:math-ph/0603010, 2006.

[36] M. G. Krĕn. Solution of the inverse Sturm-Liouville problem. (Russian) Doklady Akad. Nauk SSSR (N.S.) 76:21-24, 1951.

[37] M. G. Krein. On the transfer function of a one-dimensional boundary problem of the second order. (Russian) Doklady Akad. Nauk SSSR (N.S.) 88:405-408, 1953.

[38] M. G. Kreĭn. On determination of the potential of a particle from its S-function. (Russian) Dokl. Akad. Nauk SSSR (N.S.) 105:433-436, 1955.

[39] M. G. Kreĭn. The fundamental propositions of the theory of representations of Hermitian operators with deficiency index $(m, m)$. Ukrain. Mat. Žurnal, 1(2):3-66, 1949.

[40] P. D. Lax and R. S. Phillips. Scattering theory. Pure and Applied Mathematics, Vol. 26. Academic Press, New York-London, 1967.

[41] N. Levinson. The inverse Sturm-Liouville problem. Mat. Tidsskr. B. 1949:25-30, 1949.

[42] N. Levinson. A certain explicit relation between the phase shift and scattering potential Phys. Rev. 89:755-757, 1953

[43] M. S. Livshitz. On a certain class of linear operators in Hilbert space. Rec. Math. [Mat. Sbornik] N.S., 19(61):239-262, 1946.

[44] N. G. Makarov, V. I. Vasjunin. A model for noncontractions and stability of the continuous spectrum. Lecture Notes in Math., 864:365-412, 1981.

[45] V. A. Marčenko. On reconstruction of the potential energy from phases of the scattered waves. (Russian) Dokl. Akad. Nauk SSSR (N.S.) 104:695-698, 1955.

[46] V. A. Marčenko. Sturm-Liouville Operators and Applications (2nd ed.) Providence: American Mathematical Society, 2011.

[47] S. N. Naboko. Absolutely continuous spectrum of a nondissipative operator, and a functional model. I. Zap. Naučn. Sem. Leningrad. Otdel Mat. Inst. Steklov. (LOMI), 65:90-102, 204-205, 1976. Investigations on linear operators and the theory of functions, VII.

[48] S. N. Naboko. Functional model of perturbation theory and its applications to scattering theory. Trudy Mat. Inst. Steklov., 147:86-114, 203, 1980. Boundary Value Problems of Mathematical Physics, 10.

[49] S. N. Naboko. Nontangential boundary values of operator $R$-functions in a half-plane. Algebra i Analiz, 1(5):197-222, 1989.

[50] S. N. Naboko. On the conditions for existence of wave operators in the nonselfadjoint case. Wave propagation. Scattering theory, Amer. Math. Soc. Transl. Ser. 2, 157:127-149, Amer. Math. Soc., Providence, RI, 1993. 
[51] B. S. Pavlov. Conditions for separation of the spectral components of a dissipative operator. Izv. Akad. Nauk SSSR Ser. Mat., 39:123-148, 240, 1975. English translation in: Math. USSR Izvestija, 9:113-137, 1975.

[52] B. S. Pavlov. Diation theory and the spectral analysis of non-selfadjoint differential operators. Proc. 7th Winter School, Drogobych, 1974, TsEMI, Moscow, 2-69, 1976. English translation: Transl., II Ser., Am. Math. Soc 115:103-142, 1981.

[53] B. S. Pavlov. Selfadjoint dilation of a dissipative Schrödinger operator, and expansion in its eigenfunction. (Russian) Mat. Sb. (N.S.) 102(144): 511-536, 631, 1977.

[54] D. B. Pearson. Conditions for the existence of the generalized wave operators. J. Mathematical Phys. 13:1490-1499, 1972.

[55] M. Reed and B. Simon. Methods of modern mathematical physics. III. Academic Press [Harcourt Brace Jovanovich Publishers], New York, 1979. Scattering theory.

[56] R. Romanov. On the concept of absolutely continuous subspace for nonselfadjoint operators. J. Operator Theory 63(2):375-388, 2010.

[57] M. Rosenblum and J. Rovnyak. Hardy classes and operator theory. Oxford Mathematical Monographs. The Clarendon Press Oxford University Press, New York, 1985. Oxford Science Publications.

[58] M. Rosenblum and J. Rovnyak. Topics in Hardy classes and univalent functions. Birkhäuser Advanced Texts: Basler Lehrbücher. [Birkhäuser Advanced Texts: Basel Textbooks]. Birkhäuser Verlag, Basel, 1994.

[59] V. Ryzhov. Absolutely continuous and singular subspaces of a nonselfadjoint operator. $J$. Math. Sci. (New York) 87(5):3886-3911, 1997.

[60] V. Ryzhov. Functional model of a class of non-selfadjoint extensions of symmetric operators. In Operator theory, analysis and mathematical physics, Oper. Theory Adv. Appl., 174:117-158. Birkhäuser, Basel, 2007.

[61] V. Ryzhov, Functional model of a closed non-selfadjoint operator. Integral Equations Operator Theory 60(4):539-571, 2008.

[62] V. Ryzhov. Spectral boundary value problems and their linear operators, 38 pp., arXiv:0904.0276; in the present volume.

[63] V. Ryzhov, Equipped absolutely continuous subspaces and the stationary construction of wave operators in nonselfadjoint scattering theory. J. Math. Sci. (New York) 85(2):1849-1866, 1997.

[64] K. Schmüdgen. Unbounded self-adjoint operators on Hilbert space, Volume 265 of Graduate Texts in Mathematics. Springer, Dordrecht, 2012.

[65] I. M. Stein Singular integrals and differentiability properties of functions. Princeton Math Ser. vol. 30, Princeton Univ. Press, Princeton, NJ, 1970.

[66] B. M. Solomyak. Scattering theory for almost unitary operators, and a functional model. J. Soviet Math. 61(2):2002-2018, 1992.

[67] B. Sz.-Nagy, C. Foias, H. Bercovici, and L. Kérchy. Harmonic Analysis of Operators on Hilbert Space. Universitext. Springer, New York, Second enlarged edition, 2010.

[68] A. S. Tikhonov. An absolutely continuous spectrum and a scattering theory for operators with spectrum on a curve, St. Petersburg Math. J. 7 (1):169-184, 1996. 
[69] J. von Neumann. Über adjungierte Funktionaloperatoren. Ann. Math. 33(2):294-310, 1932

[70] J. von Neumann. Mathematical foundations of quantum mechanics. Princeton University Press, Princeton, 1955. Translated by Robert T. Beyer.

[71] M. Rosenblum. Perturbation of the continuous spectrum and unitary equivalence. Pacific J. Math., 7:997-1010, 1957.

[72] J. Weidmann. Linear operators in Hilbert spaces, volume 68 of Graduate Texts in Mathematics. Springer-Verlag, New York, 1980. Translated from the German by Joseph Szücs.

[73] D. R. Yafaev. Mathematical scattering theory, Volume 105 of Translations of Mathematical Monographs. American Mathematical Society, Providence, RI, 1992. General theory, Translated from the Russian by J. R. Schulenberger. 